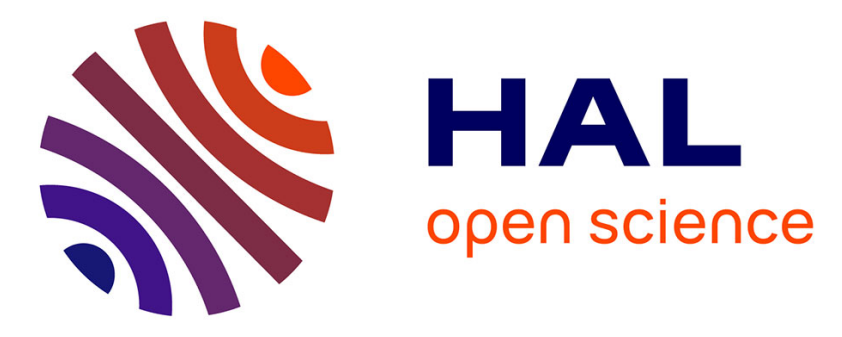

\title{
Electron energy loss spectroscopic studies of brown diamonds
}

\author{
Ursel Bangert, Rachel Barnes, Luke Hounsome, Bob Jones, Alexander \\ Thorsten Blumenau, Patrick R Briddon, Mike J Shaw, Sven Öberg
}

\section{- To cite this version:}

Ursel Bangert, Rachel Barnes, Luke Hounsome, Bob Jones, Alexander Thorsten Blumenau, et al.. Electron energy loss spectroscopic studies of brown diamonds. Philosophical Magazine, 2006, 86 (29-31), pp.4757-4779. 10.1080/14786430600776348 . hal-00513710

\author{
HAL Id: hal-00513710 \\ https://hal.science/hal-00513710
}

Submitted on 1 Sep 2010

HAL is a multi-disciplinary open access archive for the deposit and dissemination of scientific research documents, whether they are published or not. The documents may come from teaching and research institutions in France or abroad, or from public or private research centers.
L'archive ouverte pluridisciplinaire HAL, est destinée au dépôt et à la diffusion de documents scientifiques de niveau recherche, publiés ou non, émanant des établissements d'enseignement et de recherche français ou étrangers, des laboratoires publics ou privés. 


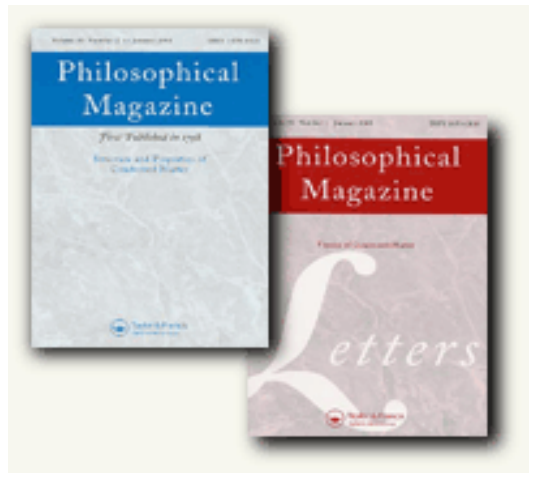

\section{Electron energy loss spectroscopic studies of brown diamonds}

\begin{tabular}{|r|l|}
\hline Journal: & Philosophical Magazine \& Philosophical Magazine Letters \\
\hline Manuscript ID: & TPHM-06-Mar-0045.R2 \\
\hline Journal Selection: & Philosophical Magazine \\
\hline Date Submitted by the & 20-Apr-2006 \\
\hline Complete List of Authors: & $\begin{array}{l}\text { Bangert, Ursel; University of Manchester, School of Materials } \\
\text { Barnes, Rachel; University of Manchester, School of Materials } \\
\text { Hounsome, Luke; University of Exeter, School of Physics } \\
\text { Jones, Bob; University of Exeter, School of Physics } \\
\text { Blumenau, Alexander; Max-Planck-Institut für Eisenforschung } \\
\text { Briddon, Patrick; University of Newcastle, School of Natural Science } \\
\text { Shaw, Mike; University of Newcastle, School of Natural Science } \\
\text { Öberg, Sven; Lulea University of Technology, Mathematics }\end{array}$ \\
\hline Keywords: & ab initio, diamond, dislocations, EELS \\
\hline Keywords (user supplied): & \multicolumn{2}{|l}{} \\
\hline hounsome_eels3.tex \\
to PDF. You must view these files (e.g. movies) online.
\end{tabular}

\section{S) ScholaroNE \\ Manuscript Central}




\title{
Electron energy loss spectroscopic studies of brown diamonds
}

\author{
U. BANGERT ${ }^{\dagger}$, R. BARNES ${ }^{\dagger}$, L. S. HOUNSOME* ${ }^{*}$, R. JONES ${ }^{\ddagger}$, A. T. BLUMENAU ${ }^{\diamond}$, \\ P. R. BRIDDON $\sharp$, M. J. SHAW ${ }^{\sharp}$ and S. ÖBERG ${ }^{b}$
}

\author{
${ }^{\dagger}$ School of Materials, University of Manchester,Manchester, M1 7HU, U.K \\ ${ }^{\ddagger}$ School of Physics, University of Exeter, Stocker Road, Exeter. EX4 4QL, U.K \\ ${ }^{\diamond}$ Max-Planck-Institut für Eisenforschung GmbH, Max-Planck-Str. 1, D-40237 Düsseldorf,Germany \\ \#Physics Centre, School of Natural Science, Newcastle upon Tyne. NE1 7RU, U.K \\ ${ }^{b}$ Department of Mathematics, Luleå University of Technology, SE-97187 Luleå, Sweden
}

We investigate both experimentally and theoretically low-loss electron energy losses in brown type IIa monocrystalline diamonds both before and after high-temperature, high-pressure anneals which remove the brown colouration. We find additional losses within and near the band edge for brown diamond which are significantly reduced after treatment. The additional losses are not associated with dislocations. Graphitic inclusions are detected by EELS as well as TEM studies for some brown diamonds before treatment. These lead to pronounced sub-gap absorption. However, all brown diamonds exhibit additional losses which are due to point defects lying in the regions between dislocations. First principles theoretical modelling shows that common dislocations are not responsible for the brown colouration but a $\pi$-bonded vacancy disk lying on $\{111\}$ planes gives broad bands lying in the diamond band gap, possesses an optical absorption spectrum similar to that of brown diamond, and leads to additional electron energy losses in the band edge region. These and similar defects are suggested to be responsible for the brown colouration. Mechanisms are proposed for their formation and removal.

Keywords: Electron Energy Loss Spectroscopy, Ab-initio, Diamond, Dislocations, Vacancy

\section{Introduction}

Low-loss electron energy loss spectroscopy (EELS) offers the possibility of revealing the local electronic structure of extended defects in semiconductors or insulators with $\sim 10 \mathrm{~nm}$ spatial resolution and with energy resolution down to $0.3 \mathrm{eV}$. Diamond is an ideal prototype material to investigate by low-loss EELS as the band gap of $5.5 \mathrm{eV}$ is large enough to minimise the influence of the zero-loss peak and any defect having gap levels is of immediate interest given the importance of diamond for the gem industry. Here we report on a joint experimental and theoretical investigation of low-loss EELS in diamond. Low-loss EELS involves measuring the loss of energy of a electron beam in promoting transitions between occupied and empty levels where the energy separation is less than about $\sim 50 \mathrm{eV}$. Core-loss EELS which is due to transitions between core levels and empty levels is much harder to investigate by modern theoretical methods as it requires the detailed knowledge of the core wavefunctions which are not available to calculations using pseudopotentials for which the core electrons are removed.

A particular problem highlighted here is the origin of brown colouration in type IIa diamonds. This has recently become of interest due to the discovery that such colouration could be removed 
through a high pressure and temperature (HPHT) treatment $[1,2,3]$. It has been proposed that the cause of this colouration is possibly a dislocation or an $\pi$-bonded extended carbon defect [4].

The characteristic absorption spectrum of brown diamond is unusual and in monocrystalline type IIa (chemically pure) diamonds, as well as diamonds grown by CVD, it follows an approximately $E^{2}$ or $E^{3}$ dependence with energies $E$ ranging from $1-5.5 \mathrm{eV}$ (eg. see Fig. 10 below). There is no evidence for an absorption edge. Additional features due to nitrogen or other defects are usually present in CVD diamond. The lack of an absorption edge could be explained if the brown absorption is due to an extended defect with broad bands of gap states, arising from an $s p^{2}$ or $\pi$-bonded defect.

In this paper we shall describe the experimental and theoretical background to low-loss EELS in section 2 and briefly review previous studies on dislocations in diamond. In section 3 we link the energy loss function with the complex dielectric constant and compare the calculation of this quantity with experiment. We then use the dielectric constant to compare the calculated and observed absorption spectra of diamond. Finally, we compare the calculated loss function with the experimental EEL intensity. In section 4 we review brown diamonds and describe EEL spectra of both brown and annealed single crystal natural type IIa diamonds in section 5. A key conclusion is that the brown colouration does not arise primarily from dislocations but from point defect clusters and graphitic inclusions. In sections 6 and 7, we describe the calculations of the absorption and loss spectra of vacancy disks and an optically active dislocation and give our conclusions in section 8 .

\section{Experimental aspects of energy loss spectroscopy}

\subsection{Development of EEL spectroscopy}

EELS has undergone constant developments since the first reports on low energy reflection EEL experiments by Rudberg in 1929 [5], and transmission energy loss measurements by Ruthemann in 1941 [6]. ELS entered the realm of microanalysis techniques, when inner shell or core losses were used for elemental analysis [7]. Through continuous improvements in instrumentation (Möllenstedt's electrostatic energy analyser, followed by retarding field spectrometer, Wien filter, Rose's Omega filter), energy analysers became incorporated into many conventional TEMs and also in STEMs. Loss spectra could be recorded as a function of position, or as a function of electron scattering angle, leading to famous studies of strong and weak losses, e.g., by Raether [8], Silcox [9] and Batson [10, 11]. Electron losses can also be displayed as a magnified image at a selected loss energy, rather then as a spectrum at a certain position (Castaing-Henry filter). EELS was further revolutionised by the use of dedicated STEMs, which, thanks to cold field emission (VG HB series) excel in brightness and energy resolution of the source. Various groups (Colliex, Brown and Howie, Spence, Batson, Pennycook) have used the VG HB5 to explore the high resolution possibilities of EELS.

EELS was pushed significantly further through the advent of position sensitive detectors, which are based on photodiode- or CCD arrays, enabling parallel rather then sequential recording. Following the development of their Model 666 spectrometer in 1986, which uses quadropole lenses to project the spectrum onto a YAG transmission screen and a photo diode array, Gatan produced the Enfina, a CCD based detector using an array of 100x1340 photodiodes. Adapted for UHV conditions, it was first installed in the VG HB6 cold field emission STEM (NorthWest STEM) at Liverpool in 2003. It is possible to conduct EELS in the loss regime of inter and intra band transitions at 0.3 
eV energy resolution without a monochromator, an option, which Bangert et al. [12] have explored in wide bandgap materials. Developments were further aided by the spectrum imaging technique, where an entire energy loss spectrum is recorded in each pixel of a pre-defined specimen area. The advantage of spectrum imaging (or mapping) over point spectrum measurements is that trends in EEL transitions in relation to positions of microstructural features can be easily seen. This also increases the possibility to hit the right spot at some position in the map, whereas even a drift of 2 Åseverely hampers point measurements on very small features. A detailed description of the spectrum imaging technique has been given by e.g., Hunt and Williams [13].

In recent developments (2003) aberration corrected lenses, developed by Krivanek [14], have been incorporated into VG STEMs (SuperSTEMs). Bleloch and Bangert have shown at the Daresbury SuperSTEM that single atom EELS (P in $\mathrm{Si}, \mathrm{Mn}$ and $\mathrm{N}$ in carbon nanotubes) is now a possibility.

\section{$2.2 \quad$ Application to dislocations}

The electronic structure of extended defects remains of great interest and has been the focus of a large body of work over the past decade, although much remains controversial. A number of EEL studies on dislocations, using core losses, have been carried out in semiconductors and wide gap materials. The fine structure of the Si-L edge revealed localised states at dislocations in SiGe [15]. Nitrogen K-edge energy-loss near-edge structure (ELNES) measurements have been performed on edge dislocations in GaN, highlighting differences from bulk regions [16]. A similar study in cubic GaN showed mid-gap levels associated with stacking faults near a GaN/GaAs interface [17]. EEL studies of the C-K edge in diamond [18] revealed increased density of states $0.5 \mathrm{eV}$ below the edge onset. Kolodzie [19] found changes in the dislocation core loss spectra in brown compared with colourless diamond. Experiments using losses at bandgap energies are rarer, due to energy resolution issues; the proximity to the tail of the zero loss peak is too high in most instruments to be able to discern the loss features. Only a handful of studies have been conducted so far: amongst those are studies of misfit dislocations at a GaAs/GaInAs interface, showing the existence of localized states at the dislocation core in GaAs [20], of dislocations in GaN, revealing pre- band edge states at the dislocation cores [21, 22] and of diamond [23]. Low-loss results of CVD diamond, interestingly, do not show a clear relationship between dislocation cores and enhanced density-ofstates (JDOS) which is a measure of available transitions, but rather an increase in $s p^{2}$ content in the vicinity of dislocations and stacking faults, suggesting existence of point defect atmospheres surrounding certain locations of extended defects. Kolodzie et al. [24] found an increase of the EEL signal in the energy region around $6 \mathrm{eV}$ at dislocations in brown diamond compared to the colourless variety. Barnes et al. [25] extended the investigations to regions remote from dislocations and found that there is a generally higher 'background' in states giving rise to intensity at $6 \mathrm{eV}$, e.g. $s p^{2}$ related defects in brown diamond than in colourless diamond.

\section{The energy loss function}

\subsection{Theory of low energy electron loss spectroscopy}

The loss of energy of a charged particle moving with speed $v$ due to ionisation of a material through which it passes, has been considered classically by Fermi [26] and quantum mechanically by Nozieres 
and Pines [27]. The rate by which energy $\hbar \omega$ and momentum $\hbar \mathbf{q}$ are lost is proportional to the imaginary part of the inverse longitudinal dielectric constant. This is called the energy loss function. Within the random phase approximation [28, 29], the expression for $\epsilon$ is

$$
\epsilon(\mathbf{q}, \omega)=1+\left(\frac{e^{2}}{\epsilon_{0} \Omega}\right) \sum_{\mathbf{k}} \frac{\left|(\mathbf{r})_{\mathbf{k} v, \mathbf{k}+\mathbf{q} c}\right|^{2}}{E_{\mathbf{k}+\mathbf{q}, c}-E_{\mathbf{k}, v}-\hbar \omega}
$$

Here $E_{\mathbf{k}, c}$ and $E_{\mathbf{k}, v}$ denote the conduction (empty) and valence (filled) bands and $\Omega$ is the volume of the crystal. $(\mathbf{r})_{\mathbf{k} v, \mathbf{k}+\mathbf{q} c}$ denotes a matrix element of the position operator with respect to an occupied valence level $\mathbf{k}, v$ and an empty level denoted by $\mathbf{k}+\mathbf{q}, c$. The constant of proportionality linking the loss intensity with the inverse dielectric constant depends weakly on energy, thus making an exact correspondence with experiment difficult. The scalar expression for the longitudinal dielectric constant is, within the random phase approximation, the same as the expression for the transverse optical dielectric constant [27] and the optical absorption coefficient, $\alpha$, is related to the complex dielectric constant by $\alpha=2 \omega \mathrm{Imag} \cdot \sqrt{\epsilon} / c$.

From a theoretical point of view, the imaginary part to $\epsilon(\mathbf{q}, \omega)$ and written as $\epsilon_{2}(\mathbf{q}, \omega)$, is first found and then its real part, $\epsilon_{1}(\mathbf{q}, \omega)$, is given by the Kramers-Kronig relation

$$
\epsilon_{1}(\mathbf{q}, \omega)=1+\frac{2}{\pi} P \int_{0}^{\infty} \frac{\omega^{\prime} \epsilon_{2}\left(\mathbf{q}, \omega^{\prime}\right)}{\omega^{\prime 2}-\omega^{2}} d \omega^{\prime} .
$$

A more general tensorial expression for the dielectric constant appropriate for extended defects is

$$
\epsilon_{i j}(\mathbf{q}, \omega)=\delta_{i j}+\left(\frac{e^{2}}{\epsilon_{0} \Omega}\right) \sum_{\mathbf{k}} \frac{(\mathbf{r} . \mathbf{i})_{\mathbf{k} v, \mathbf{k}+\mathbf{q} c}(\mathbf{r} . \mathbf{j})_{\mathbf{k}+\mathbf{q} \mathbf{c}, \mathbf{k} v}}{E_{\mathbf{k}+\mathbf{q}, c}-E_{\mathbf{k}, v}-\hbar \omega}
$$

In the case where the momentum loss $\hbar \mathbf{q}$ lies in the beam direction, then for sufficiently high energies $q=\omega / v$ can be taken to be zero. We take the average of the diagonal components of the tensor in order to compare with experimental results. This is related to the trace of the tensor and is independent of the orientation of the defect in the cell.

The dielectric constant can be evaluated from matrix elements of the Kohn-Sham wavefunctions and energy levels obtained by local density functional theory. This has been implemented in the AIMPRO code [30, 31]. The dielectric constant is sensitive to the basis used to describe the upper parts of the conduction bands. In this paper we use a minimum of 28 Cartesian Gaussian functions per atom and we have tested the sensitivity of the dielectric constant to the basis set used. For each k-point, there are a number of vertical transitions possible between occupied and unoccupied states. To achieve a relatively smooth curve, a grid of $40^{3} \mathrm{k}$-points is required for bulk diamond. Furthermore, the contribution of each transition has to be broadened over an energy interval and we use here a value of $0.8 \mathrm{eV}$.

It is important to recognise that the expression for $\epsilon$ is limited and relies on perturbation theory and the neglect of local fields, plasmons and excitonic effects. More involved theories of dielectric functions have been developed for semiconductors, to describe effects beyond the local density approximation [32], beyond the long-wavelength limit [33], and beyond the independent particle limit $[33,34]$, but they are currently too computationally intensive to be applied to anything other than bulk materials. 


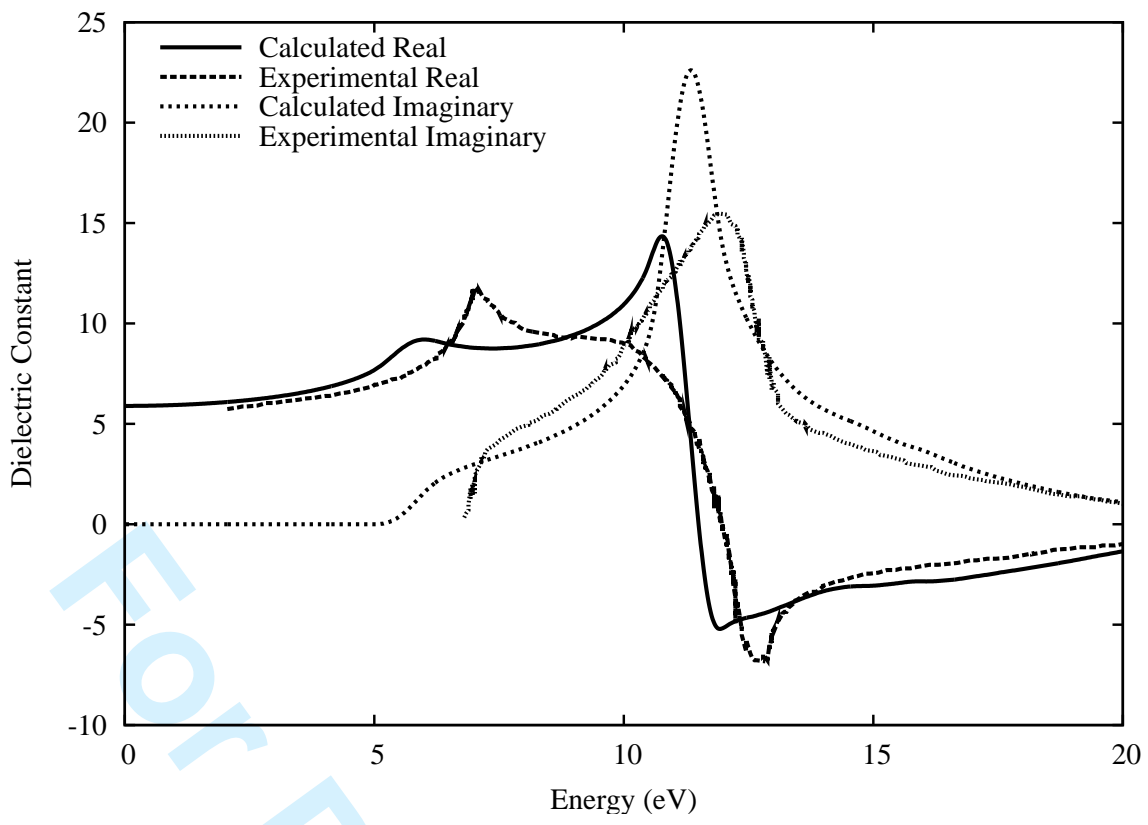

Figure 1: Real and imaginary parts of the calculated dielectric constant for bulk diamond compared to experiment. Experimental values from [35].

\subsection{Bulk diamond: dielectric constants}

Fig. 1 shows the real and imaginary parts of the dielectric constant of diamond found using a primitive two atom unitcell and compared with experimental data $[35,36]$. The experimental spectrum for $\epsilon_{2}$ shows a threshold around the direct band gap at $7 \mathrm{eV}$ and a peak at $12 \mathrm{eV}$ due to band-band transitions. It then falls slowly with increasing energy. Unusually, and in contrast with other semiconductors, diamond does not exhibit additional sharp peaks in this region due to band structure.

The theoretical curve displays a threshold at $5.4 \mathrm{eV}$, and a peak around $12 \mathrm{eV}$, which are shifted from the experimental values due to the underestimation of the gap and lower conduction bands. It is notable that when modelling diamond using AIMPRO both the direct and indirect gap can be bought into agreement with experimental values, by scaling the levels from the valence band top upwards by a factor of 1.3. This technique has been applied to the defect related gap absorption to be described later. The method of scaling energy levels is quite distinct from a rigid energy shift, also known as a 'scissors' shift. Here all levels above the valance band top have the same value shift added, but this has the effect of changing the relative position of gap levels and hence related energy transitions, so is felt unsuitable for this work. The sharp rise near $7 \mathrm{eV}$, due to excitons, is not reproduced.

The real part of the dielectric constant is also shown in Fig. 1 where it is compared with experiment. We note that in the long wave-length limit, the calculated value of 5.89 agrees well with the experimental value of the static dielectric constant of 5.82. The experimental peak at $7 \mathrm{eV}$ 
is much sharper than the calculated one due to the neglect of excitonic effects. On the other hand the energy where $\epsilon_{1}=0$ is quite accurately found.

\subsection{Bulk diamond: optical absorption and EELS spectrum}

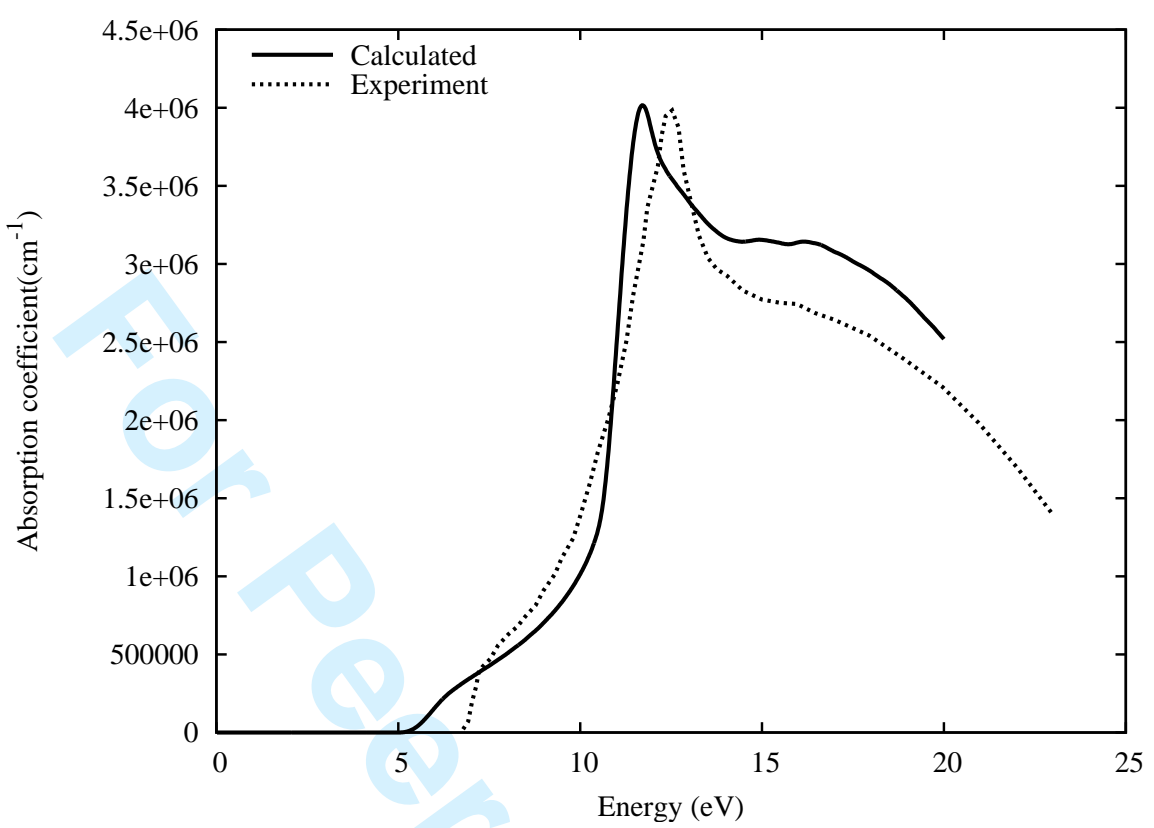

Figure 2: Calculated and experimental absorption coefficient of bulk diamond. Experimental values from [35].

The calculated and experimental absorption coefficients are shown in Fig. 2. The magnitude of the absorption agrees well with experiment but the threshold around $7 \mathrm{eV}$ clearly reflects the underestimation of the direct gap by the theory. The peak in absorption at $\sim 12.5 \mathrm{eV}$ is related to the vanishing of $\epsilon_{1}$ and is reproduced in the theoretical spectrum. However, the sharp rise of the absorption near the threshold is not reproduced due to the lack of excitons in the theory, and the absorption is $\sim 13 \%$ too large in the region beyond the peak.

Fig. 3 shows the calculated loss spectra and experimental EEL spectra for diamond and the calculated loss spectrum for graphite. For diamond, the peak around $35 \mathrm{eV}$ is due to plasmons with frequency $\sqrt{ }\left(n e^{2} / \epsilon_{0} m\right)$ where the valence electrons with density $n$ behave collectively. Using $m=0.8 m_{e}$ gives a frequency of $35 \mathrm{eV}$ for diamond and $\sim 26 \mathrm{eV}$ for graphite. We note that the spectrum for graphite has a peak around $7 \mathrm{eV}$ which as we shall see later is also present in brown diamonds but is not seen following the HPHT treatment.

Fig. 4(a) and (b) show the calculated loss spectra and experimental EDEL spectra of diamond in the threshold region. The former has a threshold at $5.4 \mathrm{eV}$ due to an underestimate of the direct gap while the latter has a threshold around $5 \mathrm{eV}$ reflecting the indirect gap of $5.5 \mathrm{eV}$. That the observed EEL threshold follows the indirect gap is also seen in other semiconductors [37] and 
may be an indication of surface bandstructure, indirect transitions involving phonons, or that the $e$-beam leads to loss with non-zero q, thus allowing indirect transitions. We also show in Fig. 4(a) the loss spectrum calculated from the experimental values of $\epsilon$ given in Fig. 1. This spectrum has a threshold at $\sim 7 \mathrm{eV}$ corresponding to direct transitions. All the spectra exhibit a shoulder around 6-12 eV but there is no evidence of the peak around $12 \mathrm{eV}$ apparent in the absorption coefficient. Hence the loss function of charged particles is quite different from X-ray absorption in this region.

In summary, the calculations confirm that the EEL spectra of diamond and graphitic carbon are quite different and that a shoulder appears on the spectrum of diamond around 6-12 eV. The theory accidentally gives the correct threshold because of the underestimate of the gap and the neglect of indirect transitions.

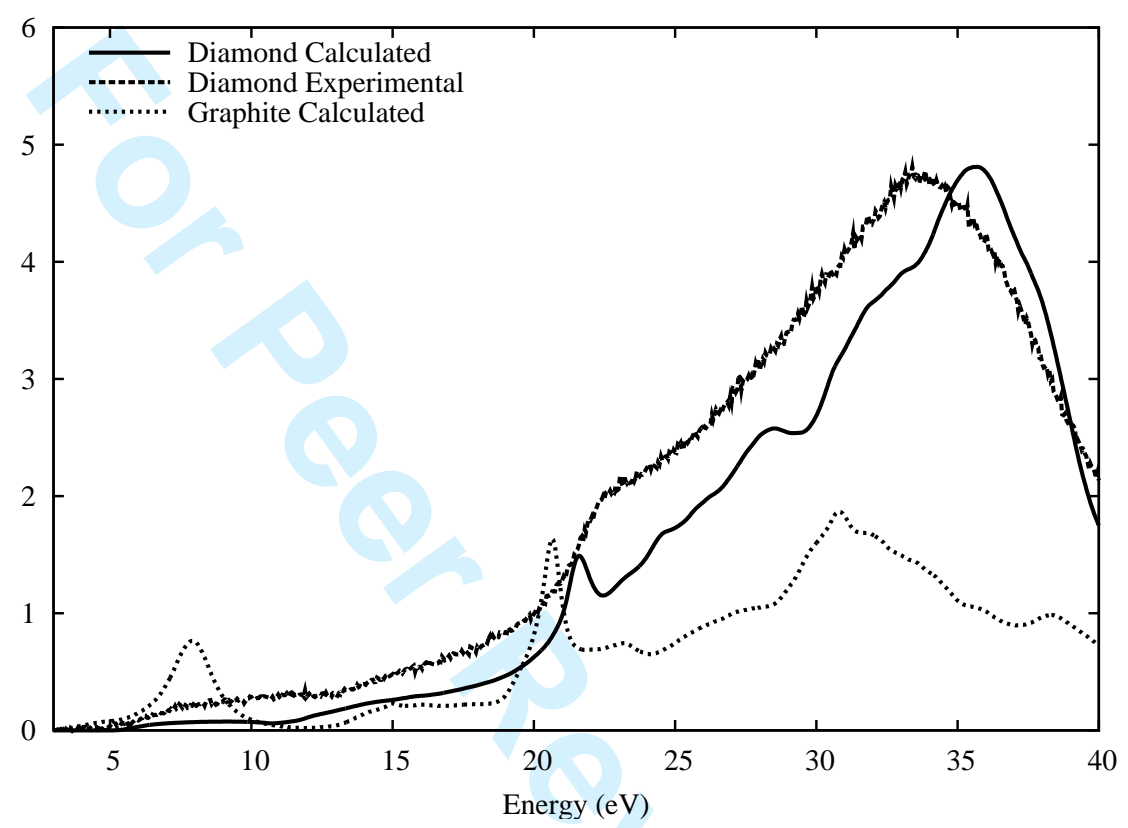

Figure 3: The calculated loss spectra found in primitive unit cells of diamond and graphite along with experimental spectrum for bulk type IIa colourless diamond. The experimental loss intensity is in arbitrary units.

\section{Introduction to brown diamonds}

The origin of the brown colour in type IIa natural and CVD diamond has been an issue of dispute for several years. Brown diamonds can be transformed into the colourless variety by High-Pressure High-Temperature (HPHT) treatment. To be able to tell whether such treatment has been applied, and, on a more fundamental level, to elucidate the origin of the colouration and the mechanism for its loss is of considerable interest to gemmologists and gem traders. Other colourations, e.g., yellow, blue, red and grey can be attributed to trace elements. However, brown type IIa diamond has few impurities and the brown colouration is largely due to native defects. 


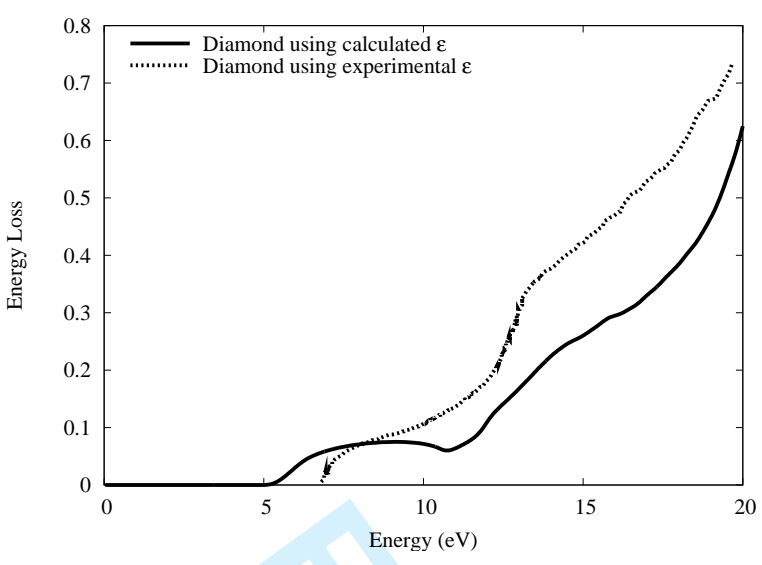

(a)

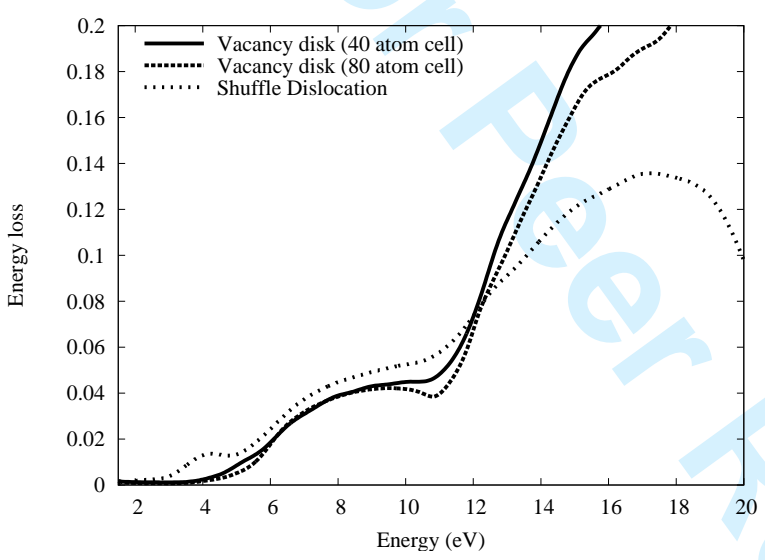

(c)

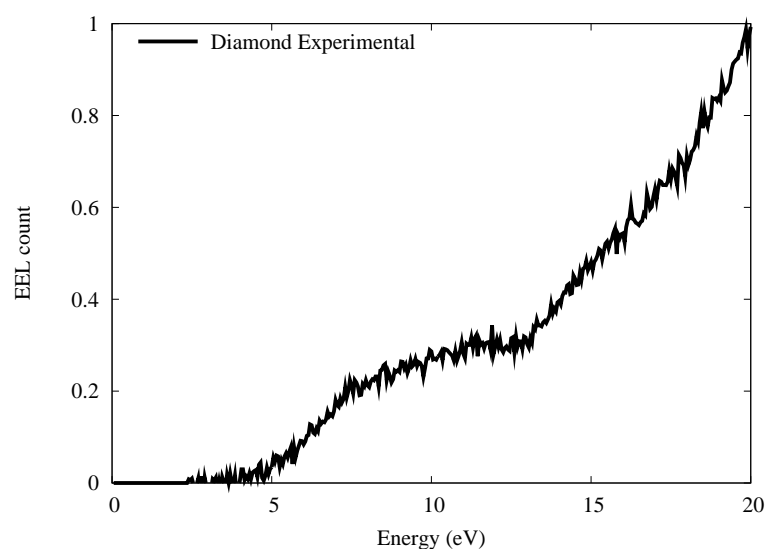

(b)

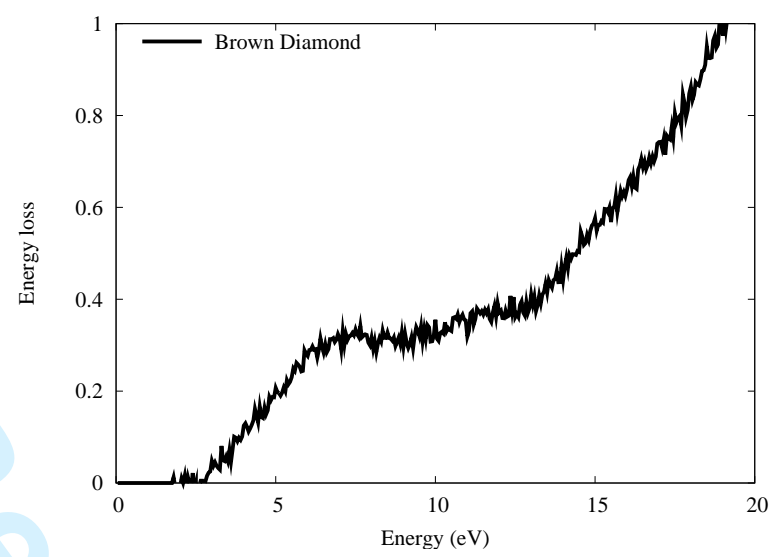

(d)

Figure 4: Calculated loss spectra and measured EEL spectra of diamond with defects. (a) Spectrum using theoretically derived $\epsilon$ and that found from experimental values of $\epsilon$. (b) Observed EEL spectrum for colourless diamond (arb. units). (c) Calculated loss spectrum for a vacancy disk in 40 and 80 atom cells, and cell containing a pair of $90^{\circ}$ shuffle and glide partial dislocations (see sections 6 and 7). (d) Observed EEL spectrum of brown diamond near graphitic region (arb. units).

It is believed that all brown natural diamonds have undergone plastic deformation at some stage in their past (although not all plastically deformed diamonds are brown), and since plastic deformation results in dislocations and there are high numbers of these in brown diamond, the question arises, in the light of lack of impurities, whether the colour is due to electronic states at dislocations. However, it is recognised that brown CVD diamonds can have very few dislocations: of density $10^{5} \mathrm{~cm}^{-2}$ compared with $10^{9} \mathrm{~cm}^{-2}$ in natural type IIa brown diamonds, and this implies that dislocations are not the sole source of brown colouration. 


\section{Experimental EELS studies of extended defects in natural single crystal brown type IIa diamonds}

The dislocation structures of brown and HPHT treated diamonds, where the brown colouration has been removed, were very similar. Investigations of the EEL spectra in spectrum maps taken of areas containing dislocations have revealed no stark differences in the spectra taken 'on' and 'off' dislocations [25]. All samples exhibited a high number of dislocations in the electron transparent area, but no other structural peculiarities. Similarly, the only microstructural features in the naturally colourless diamond were dislocations, which were similar to those in all the other diamonds, but a significant fraction of them were reticulated as in a grain-boundary-like structure [25]. In summary, there is no evidence that dislocations are the source of brown colouration.

Some brown diamonds, apart from exhibiting dislocation structures, additionally had regions of unusual diffraction contrast of 1 to $10 \mu \mathrm{m}$ in diameter and covering from $<1 \%$ to $50 \%$ (darkest brown sample) of the electron transparent area. Diffraction patterns revealed that these were of graphitic character [25]. In the middle of such patches the graphitic character often prevailed, whereas the boundaries showed the diamond diffraction pattern underlying the graphitic pattern. 
The uniaxial nature of graphite results in the dielectic tensor function $\epsilon_{i j}$ having only two components, $\epsilon^{\|}$and $\epsilon^{\perp}$ relative to the c-axis. The electron probe angle and the collection angle of the spectrometer determine the proportions of the components that contribute to the EELS spectra. The relative intensity of the $\pi^{*}$ peak with respect to the carbon K-edge changes significantly between the two components; the $\pi^{*}$ contribution is greatest for spectra acquired parallel to the c-axis [49]. This does not, however, affect our results, as we are looking for relative changes within a region containing a microstructural defect.

Since dislocations did not appear to give exceptional features in EEL spectra, we have probed the regions between dislocations, in order to find out whether there are differences between the 'bulk' of brown and colourless diamond. Correlation with the structural properties was established by looking at individual EEL spectra of corresponding locations in a variety of brown and colourless natural type IIa diamond samples.

Fig. 5(a) compares the EEL spectra of a brown diamond containing graphitized regions with those of a structurally perfect colourless sample. One notes the peaks at 25 and $7 \mathrm{eV}$ in the former similar to the calculated spectra for graphite shown in Fig. 3. This implies a connection with graphitic inclusions which are not present in a HPHT treated diamond.

Fig. 5(b) shows the 4-12 eV energy region expanded for various diamonds from colourless to brown with graphitic regions. The spectra were taken in regions between dislocations. All spectra are diamond-like, none were actually taken inside a fully graphitic region. The figure demonstrates the evolution of transitions in the bandgap region, compared to a colourless diamond reference spectrum, i.e., each displayed spectrum was obtained by subtraction of a colourless diamond spectrum, obtained from a crystallographically perfect region, from the spectrum obtained in a defective region. The bottom spectrum is the difference between two colourless diamond spectra and scatters around the zero line, as expected. It is included to show the measurement statistics. Its intensity is surpassed in the spectra taken away from and near graphitic regions in brown diamond (solid and dash-dotted lines). A perfect diamond shows a threshold in the EEL intensity around $5.5 \mathrm{eV}$, and since the extraction of the low loss signal becomes quite inaccurate towards $2 \mathrm{eV}$, there might be low losses in the brown diamonds, which we cannot directly extract. We note, however, that the occurrence of a monotonously declining tail below $6 \mathrm{eV}$, as in spectra of brown and HPHT-treated diamonds (but not in colourless diamond), might be an indication for transitions even below $2 \mathrm{eV}$. Hence we take the intensity at $4-6 \mathrm{eV}$ as a measure for transitions into states in the bandgap. We observe that the bandgap states are spread between dislocations and are especially concentrated in the neighbourhood of graphitized regions, and they do not depend on the presence of dislocations. The EEL spectra in the $6 \mathrm{eV}$ region is consistent with that of $s p^{2}$ related transitions, however, the intensity distribution in Fig. 5(b) is rather featureless, and all brown diamonds, with graphitic regions or none, exhibit increased intensity in this regime. Hence other point defect configurations, in particular those exhibiting partially $s p^{2}$ character, cannot be ruled out. We also note that the 4-6 eV intensity of structurally perfect regions in the HPHT treated brown diamond, which has lost its brown colouration, is generally higher than in colourless diamond. We did however not observe graphitic regions in this, or any other sample which had been treated. This suggests that some point defects survive the anneal. This is supported by results from positron annihilation studies, in which the vacancy cluster concentration was found to decrease, but not to disappear completely, in high temperature anneals [46].

It is instructive to visualise the spatial distribution of the low loss (bandgap) intensity in relation 


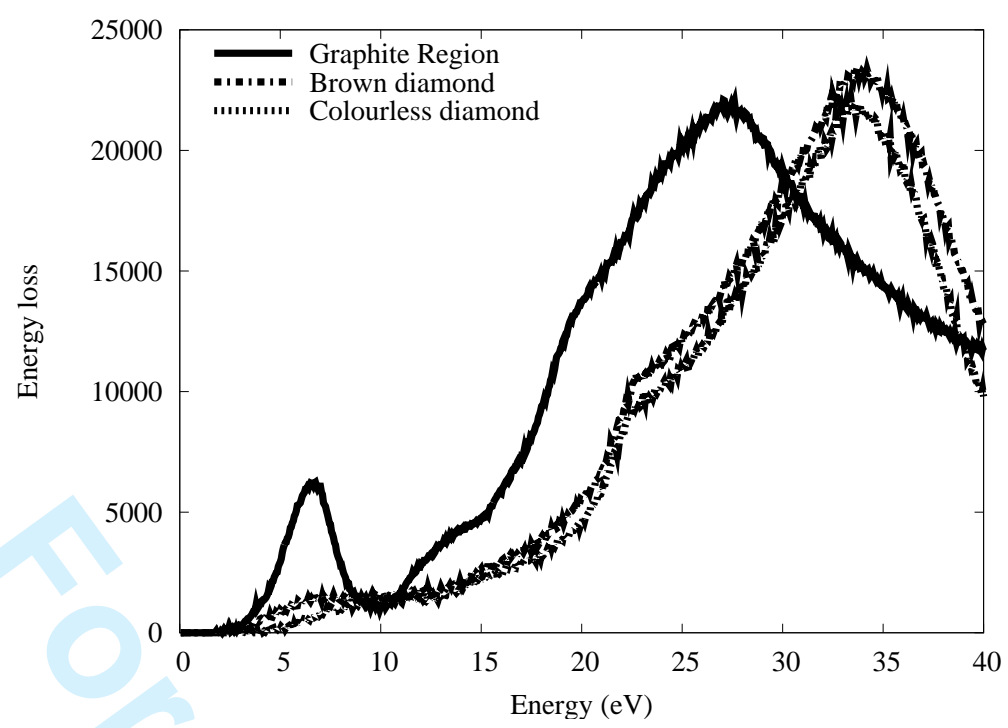

(a)

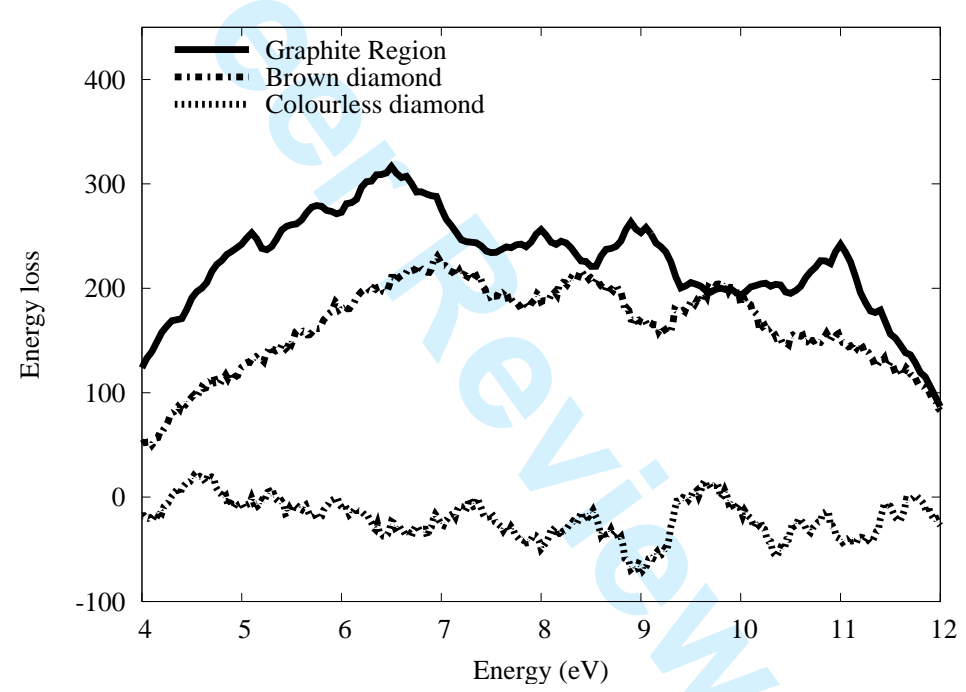

(b)

Figure 5: (a) Raw EEL spectra of colourless diamond (dashed), brown diamond at fringes of graphitized region (dash-dotted) and within graphitic region (solid). (b) EEL difference spectra, expanded at 4-12 eV, of colourless diamond (dashed), brown diamond remote from graphitic regions (dashdotted), brown diamond $100 \mathrm{~nm}$ away from graphitic region (solid).

to the microstructure. In Fig. 6, we display bright field STEM images with overlaid normalised 


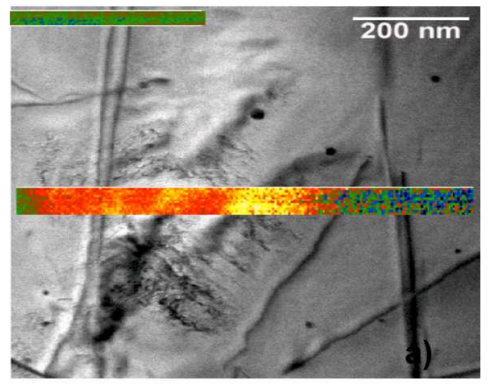

(a)

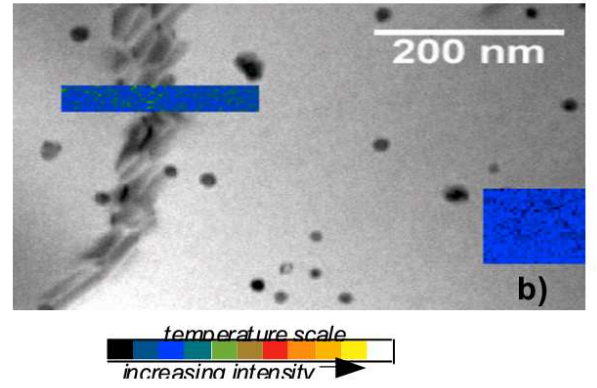

(b)

Figure 6: (a) BF image of dislocations and graphitic slivers at the fringes of graphitic regions in brown diamond. Pixel maps of the loss intensity at 4-6 eV are overlaid on the BF image in positions, in which EEL spectrum maps were obtained. (b) BF image of grain boundary in colourless diamond, with overlaid pixel maps of the loss intensity at 4-6 eV. The black dots in each image are copper contaminations originating from the microscopy support grid.
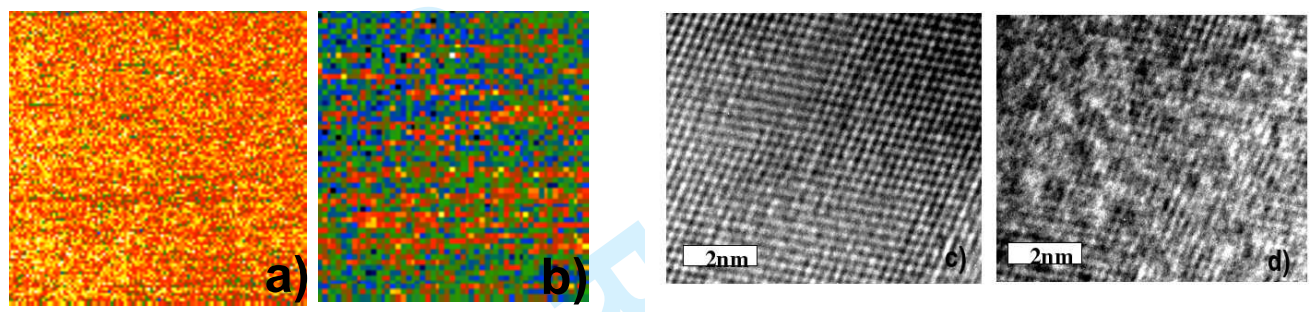

Figure 7: Maps of the relative EEL intensity at 4-6 eV, taken in non-dislocated regions in (a) brown and (b) colourless zones in a zebra sample. Also shown are lattice images taken in a TEM from (c) colourless and (d) brown zones respectively, in the same sample.

intensity maps of the integrated loss intensity in the energy window 4-6 eV. These intensity pixel maps were extracted from spectrum maps taken in the areas marked in the images. The intensities are displayed on a temperature scale, where blue indicates low, red medium and yellowish-white high intensity. The spectrum maps in Fig. 6 were taken under similar experimental conditions, and their intensities are scaled with respect to each other, so their intensity values are directly comparable. Maps in a) and b) were taken over regions including extensive material away or in between dislocations. In general it appeared that the intensity is predominantly influenced by the proximity of the graphitized regions, much more than by dislocations, hence the maps present the general 'background' intensity of the $s p^{2}$ related bond content of the respective diamond. The loss intensity at 4-6 eV shown in Fig. 6(a), typical of untreated brown diamond, varies widely across different microstructural regions. It is of a similarly low (bluish-green) value as in HPHT treated brown diamonds (not shown) when measured in locations remote from graphitic regions. Within a 
few hundred nm's of graphitic regions it increases to the levels shown in Fig. 6(a) (at the top). It increases strongly in graphitic slivers (Fig. 6(a), pixel map across the middle of image). The mottled or 'veined' patch between the dislocations in the BF image, denoted as 'sliver', is believed to arise due to few incorporated graphitic planes, which extend out from the heavily graphitic regions. In stark contrast to this is the low loss intensity in the colourless diamond: Fig. 6(b) shows part of a region of intense dislocation density with an overlaid pixel map of the losses at 4-6 eV, as well as a pixel map of non-dislocated material. It seems that overall in colourless diamond, the $s p^{2}$ background is far lower (dark blue intensity values); there are only few green pixels in the region of high dislocation density. The EEL intensity maps are all self-normalised, i.e., each spectrum intensity at $4-6 \mathrm{eV}$ is divided by the respective intensity at $12-14 \mathrm{eV}[23]$.

The clearest evidence of an increase of $s p^{2}$ content in brown regions comes from EEL intensity maps of a 'zebra' diamond (Fig. 7) [50]. The 'zebra' diamond sample is unique in containing both colourless and brown zones, separated from each other, in the same crystal, albeit without visible graphitized regions. The colour zones are arranged in roughly parallel striations. These different colour zones were identified from birefringence measurements in optical transmission micrographs taken with a cross polariser. Particular regions in the optical images, e.g., boundaries between brown and colourless zones, could unambiguously be identified and assigned to regions in the electron micrographs. The intensity maps in Figs. 7(a) and 7(b) were taken in regions of identical thickness on either side of such a boundary. The intensities have the same scales, i.e., are directly comparable (although they are not on the same scale with Figs. 6). Measurements in Fig. 7 give a direct comparison, as the spectrum images were collected in the same session within minutes of each other, i.e., in the same sample under identical instrument conditions. The maps clearly show an enhancement of EEL intensity in the 4-6 eV region in the brown sections indicating an increase of $s p^{2}$ or $\pi$-bonded defects there.

Figs. 7(c) and 7(d) show HREM lattice images of the same colourless and brown zones respectively, taken in a Tecnai F30 TEM. At high negative defocus, stark differences in the images can be seen: the brown zone exhibits contrast patches in agreement with contrast simulations of vacancy clusters in diamond [51]. The clear zone, on the other hand, shows a smooth lattice image without contrast patches.

\section{Theoretical modelling of prismatic vacancy disks}

The observations based on EELS discussed above, and the results of the positron annihilation experiments, suggest that vacancy centres might contribute to the brown colouration. We now describe a defect which contains $\pi$-bonds and has an absorption and loss spectrum similar to those observed in brown diamonds. This is the infinite planar vacancy disc.

The infinite vacancy disk is created by removing two planes of atoms as shown in Fig. 8. These lie on $\alpha B$ planes of the normal $A \alpha B \beta C \gamma$ (111) stacking sequence of diamond; their removal leads to dangling bonds lying parallel to [111] on each internal surface. The collapse of this vacancy disk, when the two planes fuse, does not lead to an intrinsic stacking fault as this requires the removal of $A \alpha$ planes. For the $\alpha B$ disk, fusing the two surfaces could be accompanied by a $a / 2(110)$ shear and this leads to a perfect dislocation loop with this Burgers vector.

The formation energy per vacancy is calculated, using either 40 or 80 atom cells, to be only 

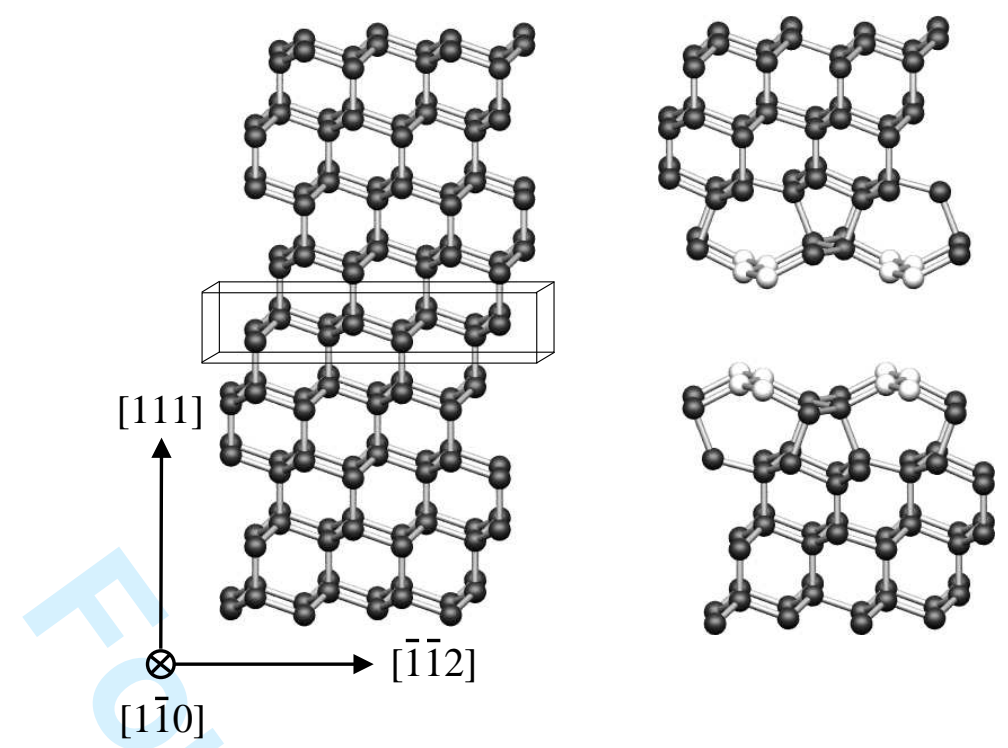

Figure 8: Illustration of the removal of a $\{111\}$ double plane (boxed) in bulk diamond and the subsequent surface rebonding, leading to a vacancy disk with chains of $\pi$-bonded atoms along $\langle 1 \overline{1} 0\rangle$ shown in white for clarity.

$1.46 \mathrm{eV}$ compared with $\sim 7 \mathrm{eV}$ for an isolated vacancy. The disk formation energy is much lower than that of clusters of up to 14 vacancies [4]. The stability of the disk is due to the elimination of dangling bonds by the formation of Pandey $\pi$-bonded chains lying along [110] [52].

The band structure of the disk, shown in Fig. 9, demonstrates that the band gap is completely filled with states and that the top of the two (due to the presence of two surfaces) occupied $\pi$ bands is degenerate with the bottom of the empty $\pi^{*}$-bands. Thus it can be anticipated that the absorption will be continuous without any threshold. The dielectric tensor for the infinite planar defect was found along the [111], $[1 \overline{1} 0]$ and $[2 \overline{1} \overline{1}]$ directions. The corresponding optical absorption coefficient, averaged over the three principal directions, is shown in a log-log plot in Fig. 10 together with experimental absorption spectra of brown natural and CVD diamonds [48]. We note that the calculated absorption is featureless in the range 1 to $5 \mathrm{eV}$, as indeed is the experimental spectrum, and the energy dependence is very similar to the absorption for the more transparent CVD-1 sample in the mid-gap region.

The expression for the dielectric constant takes only vertical electron-hole transitions into account and hence the large rise in absorption at $5.5 \mathrm{eV}$, due to indirect excitations and exciton effects, is not reproduced. In addition, the semi-metallic character of the infinite vacancy disk results in an absorption coefficient diverging at low energies [53]. A finite disk would possess a small gap between $\pi$ and $\pi^{*}$ states leading to an absorption coefficient which vanishes with energy.

The mid-gap absorption in a 40 atom cell also containing two vacancies was approximately twice that found in the 80 atom cell. This implies that the absorption in the gap region is linear in the defect density. The 80 atom cell containing two vacancies has absorption of $1447 \mathrm{~cm}^{-1}$ and thus implies that a vacancy concentration of about $2 \mathrm{ppm}\left(3 \times 10^{17} \mathrm{~cm}^{-3}\right)$ is required for an absorption 


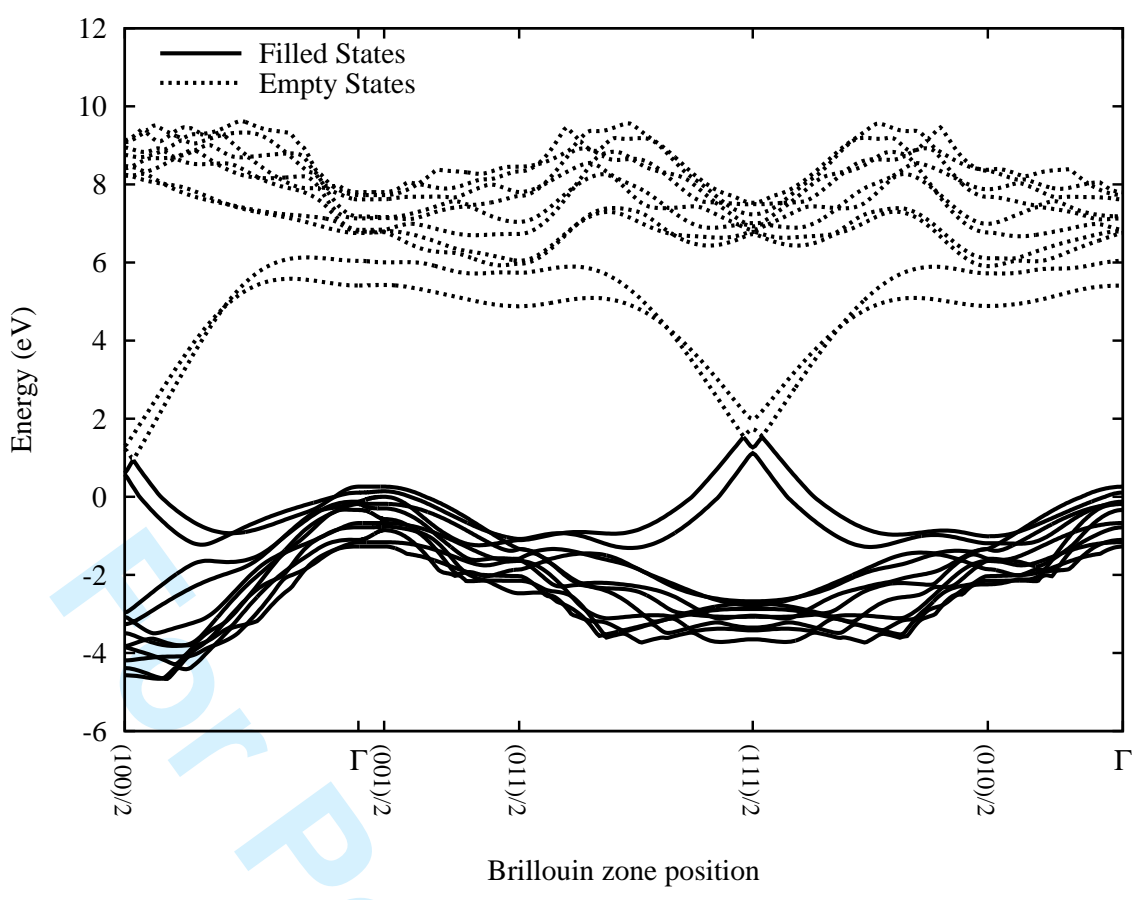

Figure 9: Band structure of the vacancy disk in an 40 atom cell. The valance band top at $\Gamma$ is set to $0 \mathrm{eV}$ and the higher energy levels are scaled by 1.3 as described in the text. Note the absence of any pronounced gap in the spectrum suggesting a featureless absorption spectrum. Dashed lines are unoccupied levels, while solid lines show filled levels.

of $0.1 \mathrm{~cm}^{-1}$ found in the natural type IIa and CVD-1 samples shown in Fig. 10.

The calculated loss spectra in the $3-20 \mathrm{eV}$ range for the two vacancy disks are shown in figure 4 where they can be compared with the experimental EEL spectrum of a brown diamond away from graphitic inclusions. The intensity increases with vacancy concentration, throughout the energy range, supporting the assumption made in section 5 that the EEL intensity in the 4- 6 eV region reflects the density of band gap levels which contribute to the absorption. This suggests that such disks are likely candidates for the centres responsible for the brown colouration.

If the vacancy disks contribute to the source of brown colour in natural type IIa and CVD diamond, we must consider the mechanism for their growth and annealing under HPHT treatments $[1,2,3]$. In natural diamonds it is possible that the disks grow through vacancy clustering where vacancies are formed by dislocation climb. Large vacancy clusters have been detected in plastically deformed Si [54]. In CVD diamond we have to suppose that disks are formed during growth. Larger graphitic regions, detected in some brown diamonds, would be stabilised by removing $2 n+2$ atomic planes $\alpha B \beta C \ldots$ and inserting $n$ graphite planes. Natural diamonds with graphitic inclusions having this orientation have been reported [55] and the graphitic inclusions discussed in section 5 probably have similar structure [25].

The mechanisms for the loss of the disks in natural and CVD diamonds would have to be 
different since the anneal conditions are different. The loss of the brown colour occurs at 1400$1600^{\circ} \mathrm{C}$ in CVD [56], and $\sim 2200^{\circ} \mathrm{C}$ in natural type IIa diamonds [57]. Now large disks would be unstable against collapse to dislocation loops. The energy of a collapsed disk of radius $r$, according to elasticity theory [60], is

$$
\sim \frac{\mu b^{2} r}{2(1-\nu)}(\ln (4 r / b)-1)
$$

Here $\mu, \nu$ and $b$ are the shear modulus, Poisson ratio and Burgers vector. This energy is less than that of the vacancy disk for loops of radius greater than about $12 \AA$ and containing about 200 vacancies. Thus we suppose in natural diamonds, small vacancy disks would dissolve leading to larger disks which collapse when their radius exceeds about $12 \AA$. The temperature when vacancies boil off of small disks can be crudely estimated by assuming that the rate of this process is given by $\nu \exp (-(B+W) / k T)$, where $\nu$ is an atomic jump frequency, taken to be $10^{13} \mathrm{~s}^{-1}, B$ is the binding energy of a vacancy with the disc which is approximately the difference in the formation energies 
of an isolated vacancy $(\sim 6 \mathrm{eV}[58])$ and a vacancy in the disc $(1.46 \mathrm{eV})$, and $W \sim 2 \mathrm{eV}$ is the migration energy of the vacancy [59]. This rate is about $1 \mathrm{~s}^{-1}$ at $2400^{\circ} \mathrm{C}$ which is in reasonable agreement with observations that the loss of the brown colour occurs around $2200^{\circ} \mathrm{C}$ [57].

Hydrogen is an important impurity in CVD diamonds and we suggest it plays a role in the removal of the brown colouration. Calculations [48] demonstrate that the hydrogen passivated disc, where each surface is stabilised by the presence of $\mathrm{C}-\mathrm{H}$ bonds, would be optically inert. It is known that when CVD brown diamonds are annealed for one hour at $1900^{\circ} \mathrm{C}$ there is a considerable loss to the brown colour and a growth of broad infra-red bands around 1331 and $2834 \mathrm{~cm}^{-1}$ as well as other modes attributed to $\mathrm{C}-\mathrm{H}$ bonds [61]. These frequencies correspond with $\mathrm{C}-\mathrm{H}$ bend and stretch modes on a (111) surface [62]. It is natural then to suppose that, during the anneal, hydrogen moves from some reservoir in the CVD diamond and passivates the disk. These different mechanisms for the removal of the brown colouration in natural and CVD diamonds would account for the different temperatures where colourless natural and CVD diamonds are formed.

\section{Theoretical modelling of glide and shuffle dislocations}

The structure and properties of $60^{\circ}$ dislocations have been reviewed previously [31, 38]. These dislocations are known to be dissociated [38] into $90^{\circ}$ and $30^{\circ}$ partials lying along [1ํㅣ], and the simplest are periodic with a repeat distance of $a / \sqrt{ } 2$. Some possess a bandstructure which could be consistent with a broad absorption. These shuffle type dislocations have unreconstructed bonds along their cores, whereas the glide types have only reconstructed bonds. The absorption and relative stability of such dislocations are now described for two types of partial dislocation. To model these types, two unitcells were constructed. In the first, a pair of glide partials is inserted along with a stacking fault of $10.9 \AA$ (Fig. 11). This is about $1 / 3$ of observed stacking fault widths. Computational limitations prevent the analysis of a more realistic stacking fault width. The cell contains 4 core atoms and 440 atoms in total.

For the second cell, atoms lying in the core of one of the partials are removed, leading to a shuffle core and a unitcell of 438 atoms (Fig. 11). The advantage of using a mixed pair is that since the glide partial does not lead to deep gap states, the electronic levels lying in the gap will only arise from the shuffle partial.

The formation energy, per core atom, of the cell containing the shuffle dislocation exceeds that containing the glide partial by $14 \mathrm{eV}$ for each core atom lying along the line. This demonstrates the greatly increased stability for the $90^{\circ}$ glide dislocation, indicating that many of the dislocations in diamond are likely to be of the glide type. However, the shuffle type could arise as a grown-in defect or when vacancies are present and are trapped at the dislocation core.

The bandstructures of the two cells are very different with the shuffle leading to mid-gap states as found previously [31]. We cannot calculate the absorption spectrum of an isolated shuffle partial as it is tied to its glide partner in the cell. However, the absorption spectrum of a pair of glide partials has a threshold close to the band gap of bulk diamond and hence the sub-gap absorption shown in Fig. 10 reflects that of the shuffle partial. This is largely featureless, much like that of brown diamond. We can estimate the required number of core atoms to produce an absorption comparable to that seen experimentally. The absorption at $2.5 \mathrm{eV}$ due to the 438 atom cell containing 2 core atoms of a shuffle dislocation is $5030 \mathrm{~cm}^{-1}$. Hence, for $0.1 \mathrm{~cm}^{-1}$ absorption, we require $2 \times 10^{16}$ 

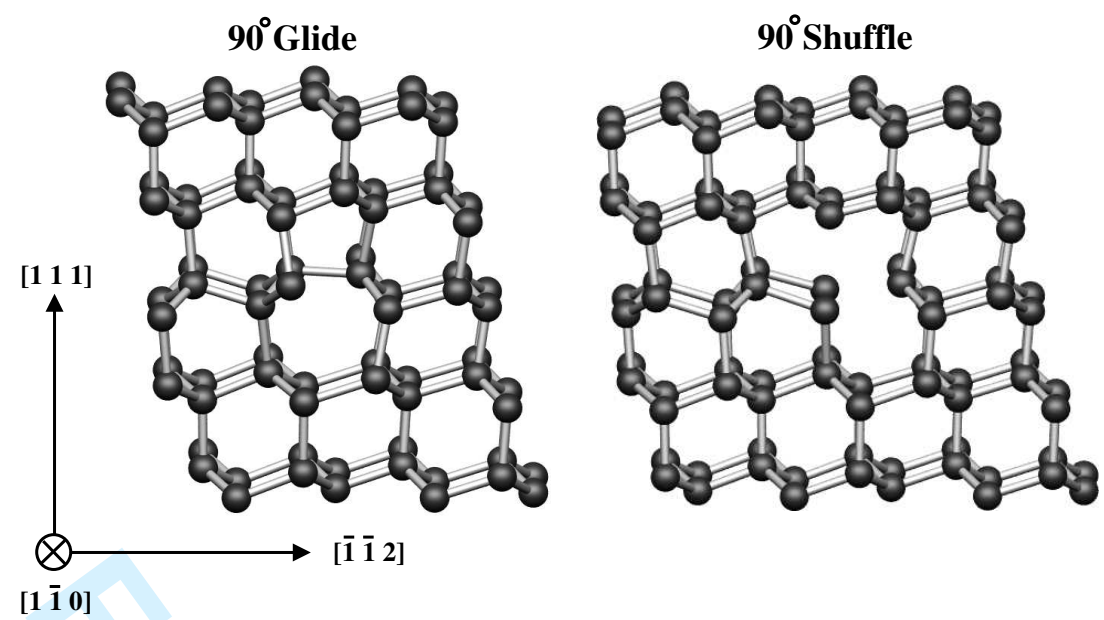

Figure 11: Structure of the $90^{\circ}$ glide (single period) and $90^{\circ}$ shuffle partial dislocation. The dislo-

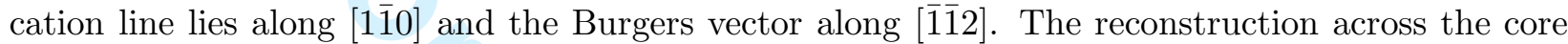
can be seen in the former but is absent in the latter

core atoms $/ \mathrm{cm}^{3}$ or a shuffle dislocation density of $N_{d}=6 \times 10^{8} \mathrm{~cm}^{-2}$. Estimates of the dislocation density found in single crystal natural type IIa brown diamonds by TEM studies [44], are about $10^{9} \mathrm{~cm}^{-2}$ both before and after the HPHT treatment to remove the colouration. Thus we require a significant fraction of the dislocations in brown diamonds to be in the shuffle form, which seems unlikely. In CVD brown single crystalline diamonds, the dislocation density can be very much lower around $10^{5} \mathrm{~cm}^{-2}[63]$ and dislocations can certainly be ruled out as the principal cause of the brown colouration. Further support for the view that dislocations are not responsible for the brown colouration comes from EELS discussed in section5.

\section{Conclusions}

The EELS observations have revealed that in type IIa diamonds, dislocations do not lead to additional loss either in the brown or HTHP treated colourless diamond. This is consistent with the theoretical finding that most dislocations are in the glide set and have only reconstructed core bonds without deep gap levels. The modelling studies indicate that shuffle dislocations can possess deep gap levels, leading to a similar absorption pattern, as seen experimentally. These however are less stable than glide dislocations and most of the dislocations found in natural diamonds would have to be of the shuffle sort to produce the required absorption intensity. Moreover, in CVD brown diamonds, dislocations are too rare to account for the measured absorption. Thus is can be said that dislocations cannot be a major source of brown absorption in both natural type IIa and CVD diamonds.

In addition to dislocations, some brown diamonds possess graphitic features, of spatial extent $\sim 200 \mathrm{~nm}$, visible in TEM and EELS. In EELS, the specific peaks in the EEL spectrum due to $\pi$ bonded carbon are detected. These graphitic inclusions may lie on $\{111\}$ planes where the interface 
is stabilised by the formation of $\pi$-bonded chains (see Fig. 8) between the graphite and diamond.

In all brown diamonds investigated, additional loss was found in the $6 \mathrm{eV}$ region which probably extends into the gap region accounting for the colour. This was clearly demonstrated in the 'zebra' diamonds, in which brown zones exhibit overall higher loss intensity in the $6 \mathrm{eV}$ regime and below. This loss intensity is particularly strong in the vicinity of graphitized regions although it is generally significantly higher then in colourless diamond.

It is not completely clear what these defects are but the theoretical modelling, together with the positron annihilation data and HREM images, indicates that multi-vacancy defects are responsible. The theoretical modelling of $\{111\}$ vacancy disks demonstrated that these possess deep gap states and lead to a featureless absorption spectrum, without a threshold, as indeed is found in brown diamonds. Further, they exhibit an enhanced loss in the $6 \mathrm{eV}$ region (Fig. 4) If the absorption is due to the disks, then an absorption coefficient of about $0.1 \mathrm{~cm}^{-1}$ at mid-gap suggests a vacancy concentration of $3 \times 10^{17} \mathrm{~cm}^{-3}$ and a disk concentration of about $10^{15} \mathrm{~cm}^{-3}$ if each disk were made from 100 vacancies. It is possible that the vacancy clusters form $\{111\}$ octahedral voids. Their optical properties would be similar to those of the disk as their surfaces would be expected to form similar $\pi$-bonded chains.

Following the HPHT treatment, there is a general decrease in the EEL intensity in the $6 \mathrm{eV}$ range, but it does not fall to the level of the colourless diamond. The HPHT treatment has obviously resulted in the removal of sufficient defects for the colour to disappear. In summary, it appears that dislocations are not the direct cause of the brown colour. Theoretical and experimental evidence suggests that vacancy aggregates are the likely source.

Questions concerning the formation and anneal of the vacancies in both natural and CVD diamonds remain. We suggest they are formed by climb processes in natural diamonds which explains their intimacy with dislocations, and that they anneal by growth and subsequent collapse into small dislocation loops. The temperature where small loops dissolve releasing vacancies is estimated to be $2400^{\circ} \mathrm{C}$ which is reasonably close to the observed temperature where the loss of colour occurs in natural diamonds. In CVD diamond, on the other hand, they may be formed during growth and the brown colouration removed by hydrogen passivation. Further investigations are required to pin-point the precise mechanism.

\section{Acknowledgement}

We thank DTC and the EPSRC for funding this work and to Philip Martineau and David Fisher for samples and numerous discussions.

\section{References}

[1] A. T. Collins, H. Kanda, and H. Kitawaki, Diamond and Related Mater. 9113 (2000).

[2] D. Fisher and R. A. Spits, Gems Gemol. 3642 (2000).

[3] S. S. Vagarali, S. W. Webb, W. E. Jackson, W. F. Banholzer, T. R. Anthony, and G. R. Kaplan, U.S Patent 6,692,714 Feb. 172004. 
[4] L. S. Hounsome, R. Jones, P. M. Martineau, M. J. Shaw, P. R. Briddon, S. Öberg, A. T. Blumenau, and N. Fujita, Phys. Status Solidi A 2022182 (2005).

[5] E. Rudberg, Kgl. Svenska Vetenskapsakad. Handl 71 (1929).

[6] G. Ruthemann, Naturwissenschaften 29648 (1941).

[7] J. Hillier and R. F. Baker, J. Appl. Phys. 15663 (1944).

[8] H. Raether, Excitations of plasmons and interband transitions, Volume 88 of Springer Tracts in Modern Physics. Springer Verlag, New York, (1980).

[9] J. Silcox, Scanning Electron Microscopy. Plenum Press, New York, (1979).

[10] P. E. Batson, Ultramicrosc. 501 (1993).

[11] P. E. Batson, Ultramicrosc. 5963 (1995).

[12] U. Bangert, A. J. Harvey and R. Keyse, Ultramicrosc. 68173 (1997).

[13] J. A. Hunt and D. B. Williams, Ultramicrosc. 3847 (1991).

[14] O. L. Krivanek, P. D. Nellist, N. Dellby, M. F. Murfitt, and Z. Szilagyi, Ultramicrosc. 96229 (2003).

[15] P. E. Batson, Phys. Rev. Lett. 834409 (1999).

[16] Y. Xin, E. M. James, I. Arslan, S. Sivananthan, N. D. Browning, S. J. Pennycook, F. Omnès, B. Beaumont, J.-P. Faurie, and P. Gibart, Appl. Phys. Lett. 76466 (2000).

[17] S. Nagahama, N. Iwasa, M. Senoh, T. Matsushita, Y. Sugimoto, H. Kiyoku, T. Kozaki, M. Sano, Matsumura, H. Umemoto, K. Chocho, T. Yanamoto, and T. Mukai, Phys. Status Solidi A 188 1 (2001).

[18] J. Bruley and P. E. Batson, Phys. Rev. B 409888 (1989).

[19] A. T. Kolodzie. EELS at Dislocations in Diamond. PhD thesis, Magdalene College, University of Cambridge, October 2003.

[20] P. E. Batsona, K. L. Kavanagha, C. Y. Wonga, and J. M. Woodall, Ultramicrosc. 2289 (1987).

[21] A. Gutierrez-Sosa, U. Bangert, and W. R. Flavell, J. Appl. Phys. 946639 (2003).

[22] U. Bangert, A. Gutiérrez-Sosa, A. J. Harvey, C. J. Fall, and R. Jones, Phys. Rev. B 932728 (2003).

[23] U. Bangert, A. J. Harvey, A. Papworth, M. Schreck, and F. Hoermann, Ultramicrosc. 10446 (2003).

[24] A. T. Kolodzie, M. Murfitt, and A. Bleloch, Institute Phys. Conf. Series 168247 (2001). 
[25] R. Barnes, U. Bangert, P. M. Martineau, D. Fisher, R. Jones, and L. S.Hounsome Journal Phys. Conf. Series 26157 (2006).

[26] E. Fermi, Phys. Rev. 57485 (1940).

[27] P. Nozières and D. Pines, Phys. Rev. 1131254 (1959).

[28] H. Ehrenreich and M. H. Cohen, Phys. Rev. 115786 (1959).

[29] S. L. Adler, Phys. Rev. 126413 (1962).

[30] R. Jones, B. J. Coomer, J. P. Goss, B. Hourahine, and A. Resende, Special defects in semiconducting materials, Volume 71 of Solid State Phenomena, Chapter 'The interaction of hydrogen with deep level defects in silicon', Pages 173-248. Scitech Publications Ltd., Zuerich-Uetikon, Switzerland, (2000).

[31] C. J. Fall, A. T. Blumenau, R. Jones, P. R. Briddon, T. Frauenheim, A. Gutiérrez-Sosa, U. Bangert, A. E. Mora, J. W. Steeds, and J. E. Butler, Phys. Rev. B 65205206 (2002).

[32] V. Olevano, M. Palummo, G. Onida, and R. Del Sole, Phys. Rev. B 6014224 (1999).

[33] W. R. L. Lambrecht and S. N. Rashkeev, Phys. Status Solidi B 217599 (2000).

[34] L. X. Benedict, T. Wethkamp, K. Wilmers, C. Cobet, N. Esser, E. L. Shirley, W. Richter, and M. Cardona, Solid State Commun. 112129 (1999).

[35] H. R. Philipp and E. A. Taft, Phys. Rev. 127159 (1962).

[36] R. A. Roberts and W. C. Walker, Phys. Rev. 161730 (1967).

[37] H. R. Philipp and H. Ehrenreich, Phys. Rev. 1291550 (1963).

[38] A. T. Blumenau, R. Jones, T. Frauenheim, B. Willems, O. I. Lebedev, G. Van Tendeloo, D. Fisher, and P. M. Martineau, Phys. Rev. B 68014115 (2003).

[39] J. Ruan, K. Kobashi, and W. J. Choyke, Appl. Phys. Lett. 603138 (1992).

[40] D. Takeuchi, H. Watanabe, S. Yamanaka, H. Okushi, H. Sawada, H. Ichinose, T. Sekiguchi, and K. Kajimura, Phys. Rev. B 63245328 (2001).

[41] P. L. Hanley, I. Kiflawi, and A. R. Lang, Phil. Trans. R. Soc. A284 329 (1977).

[42] I. Kiflawi and A. R. Lang, Phil. Mag. 30219 (1974).

[43] A. T. Kolodzie and A. L. Bleloch, Institute Phys. Conf. Series 179319 (2003).

[44] B. Willems, Presented at Diamond 2006, Oxford, July 2005.

[45] D. Fisher, Private communication, 2005.

[46] V. Avalos and S. Dannefaer, Physica B 34076 (2003). 
[47] K. Saarinen, Presented at Diamond 2006, Oxford, July 2005.

[48] L. S. Hounsome, R. Jones, P. M. Martineau, D. Fisher, M. J. Shaw, P. R. Briddon, and S Öberg, Phys. Rev. B 73125203 (2006).

[49] N. D. Browning, J. Yaun and L. M. Brown, Ultramicroscopy 38291 (1991).

[50] B. Willems, L. C. Nistor, C. Ghica, and G. Van Tendeloo, Presented at SBDD-X, LUC Belgium, February 2005.

[51] R. Barnes and U. Bangert, Presented at SBDD-XI, LUC Belgium, February 2006.

[52] K. C. Pandey, Phys. Rev. B 254338 (1982).

[53] T. G. Pedersen, Phys. Rev. B 67113106 (2003).

[54] H. S. Leipner, V. V. Mikhnovich, V. Bondarenko, Z. Wang, H. Gu, R. Krause-Rehberg, J. L. Demenet, and J. Rabier, Physica B 340617 (2003).

[55] J. Glinnemann, K. Kusaka, and J. W. Harris, Zeitschrift fur Kristallographie 218733 (2003).

[56] D. J. Twitchen, P. M. Martineau, and G. A. Scarsbrook, Patent Application WO 2004/022821, March 182004.

[57] R. C. Burns, D. Fisher, and R. A. Spitz, Patent Application WO 01/72406, April 042001.

[58] R. Q. Hood, P. R. C. Kent, R. J. Needs and P. R. Briddon, Phys. Rev. Lett. 91076403 (2003).

[59] S. J. Breuer and P. R. Briddon, Phys. Rev. B 516984 (1995).

[60] J. P. Hirth and J. Lothe, Theory of dislocations. Materials Science and Engineering. McGrawHill, New York, 1968.

[61] S. J. Charles, J. E. Butler, B. N. Feygelson, M. E. Newton, D. L. Carroll, J. W. Steeds, H. Darwish, C.-S. Yan, H. K. Mao, and R. J. Hemley, Phys. Status Solidi A 2012473 (2004).

[62] R. P. Chin, J. Y. Huang, Y. R. Shen, T. J. Chuang, and H. Seki, Phys. Rev. B 525985 (1995).

[63] P. M. Martineau, Private communication, 2004. 


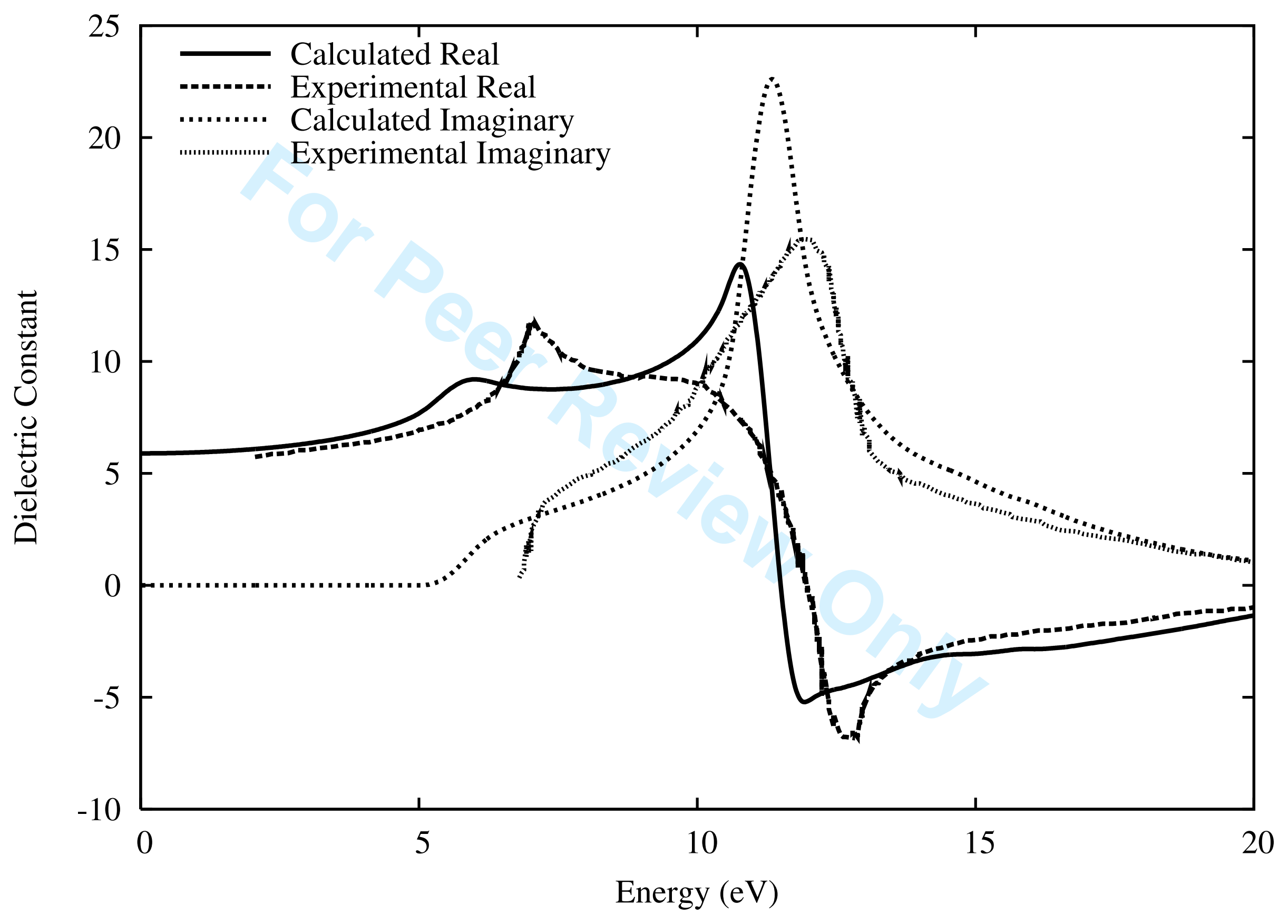

http://mc.manuscriptcentral.com/pm-pml 


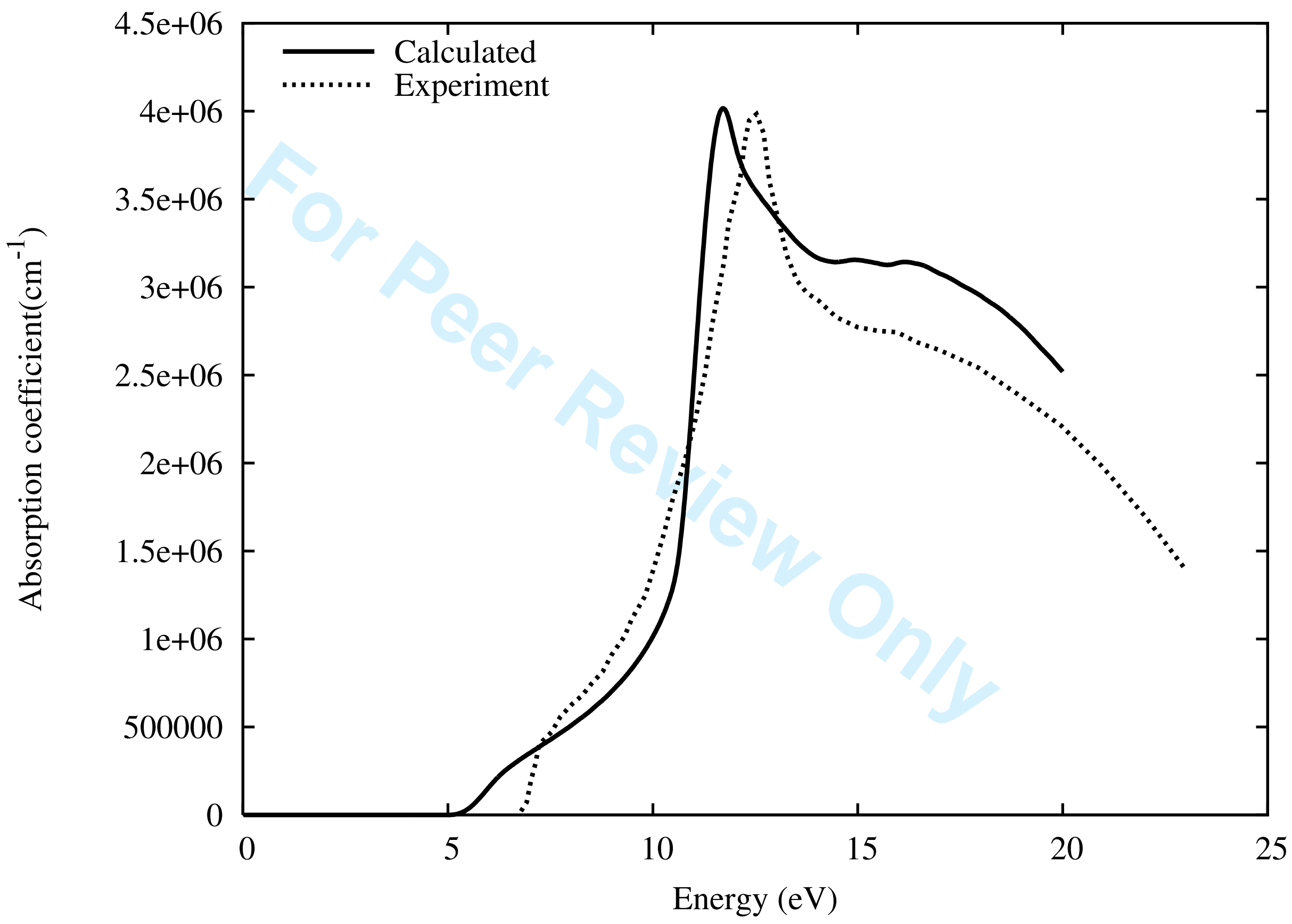

http://mc.manuscriptcentral.com/pm-pml 


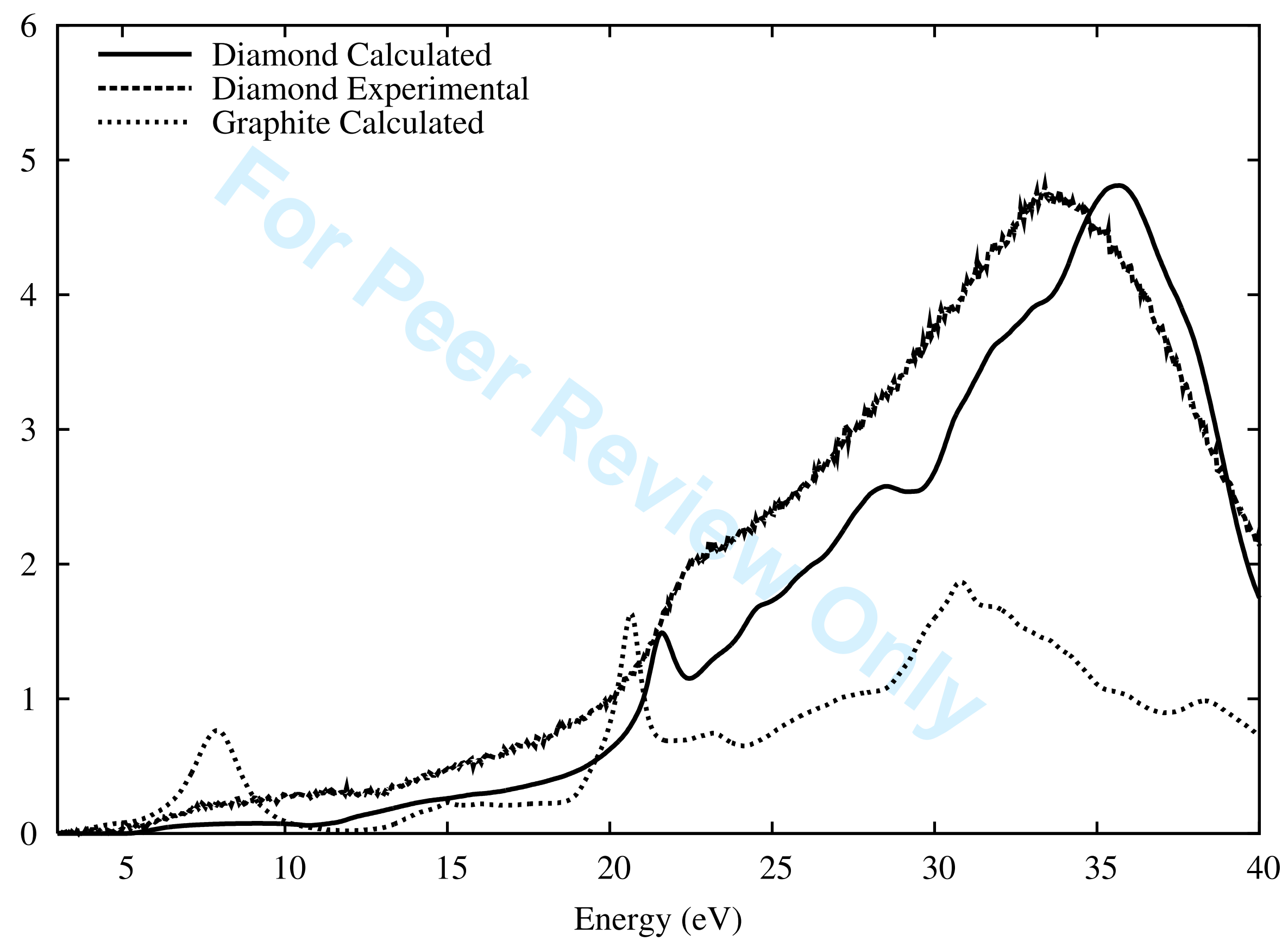

http://mc.manuscriptcentral.com/pm-pml 


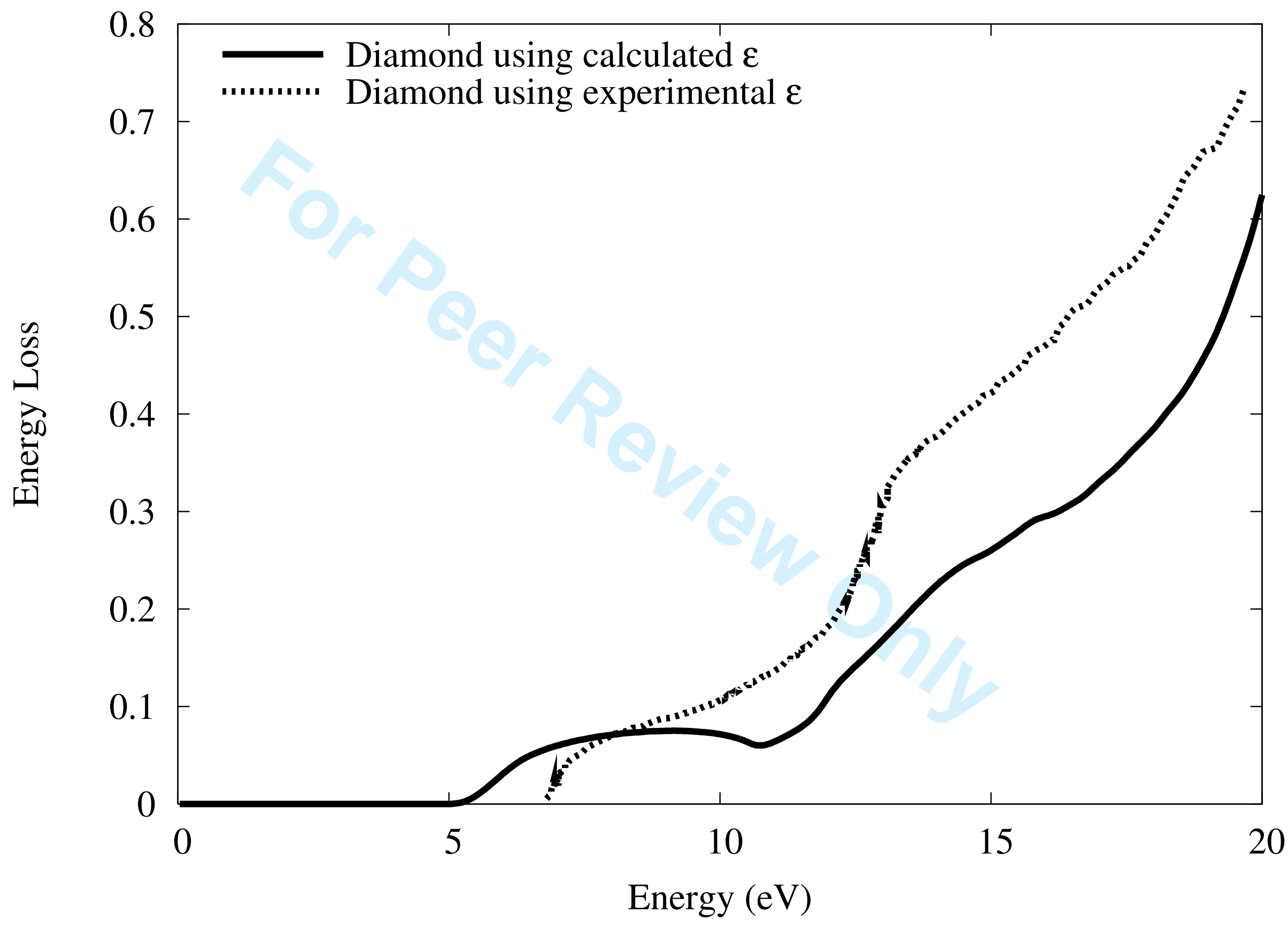

http://mc.manuscriptcentral.com/pm-pml 


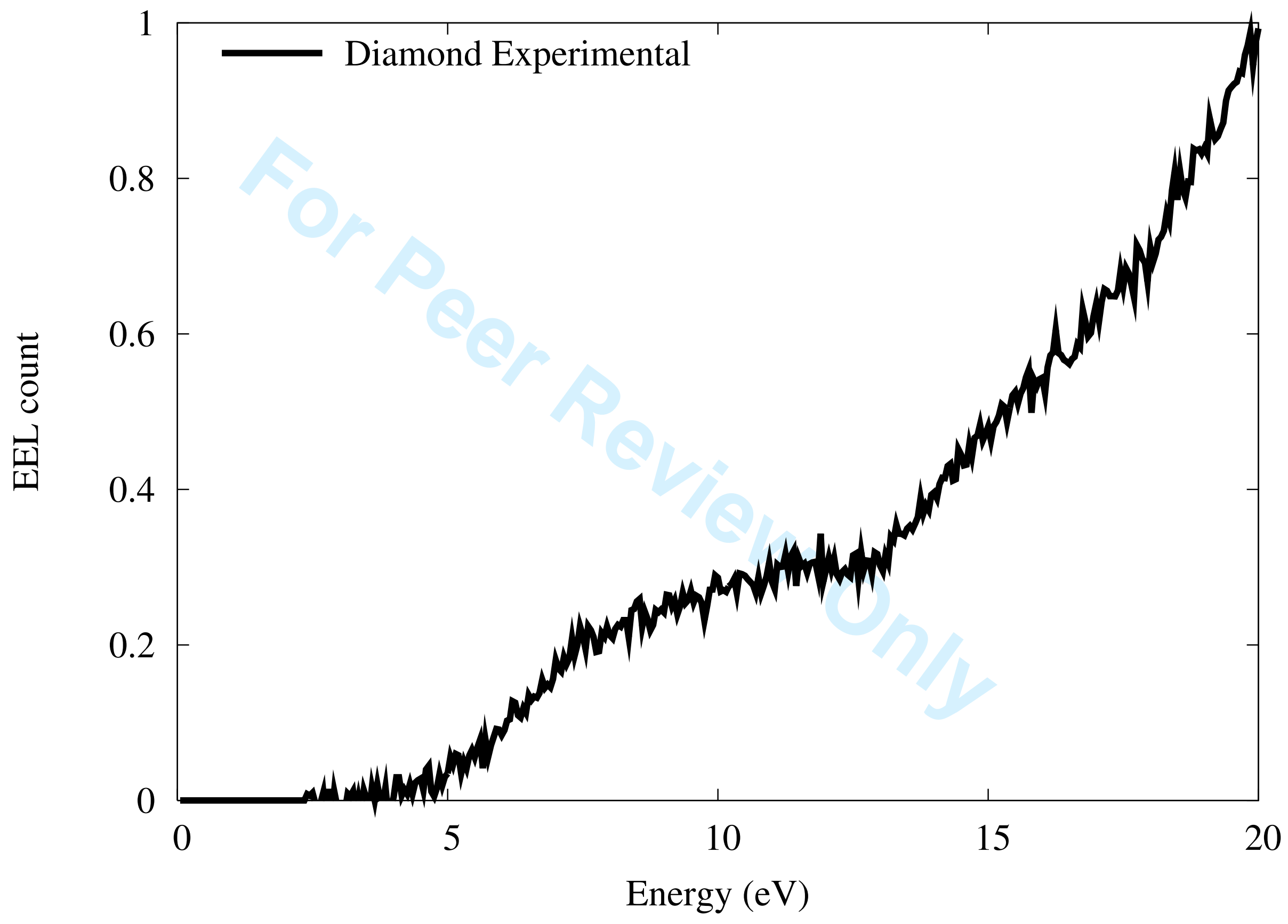




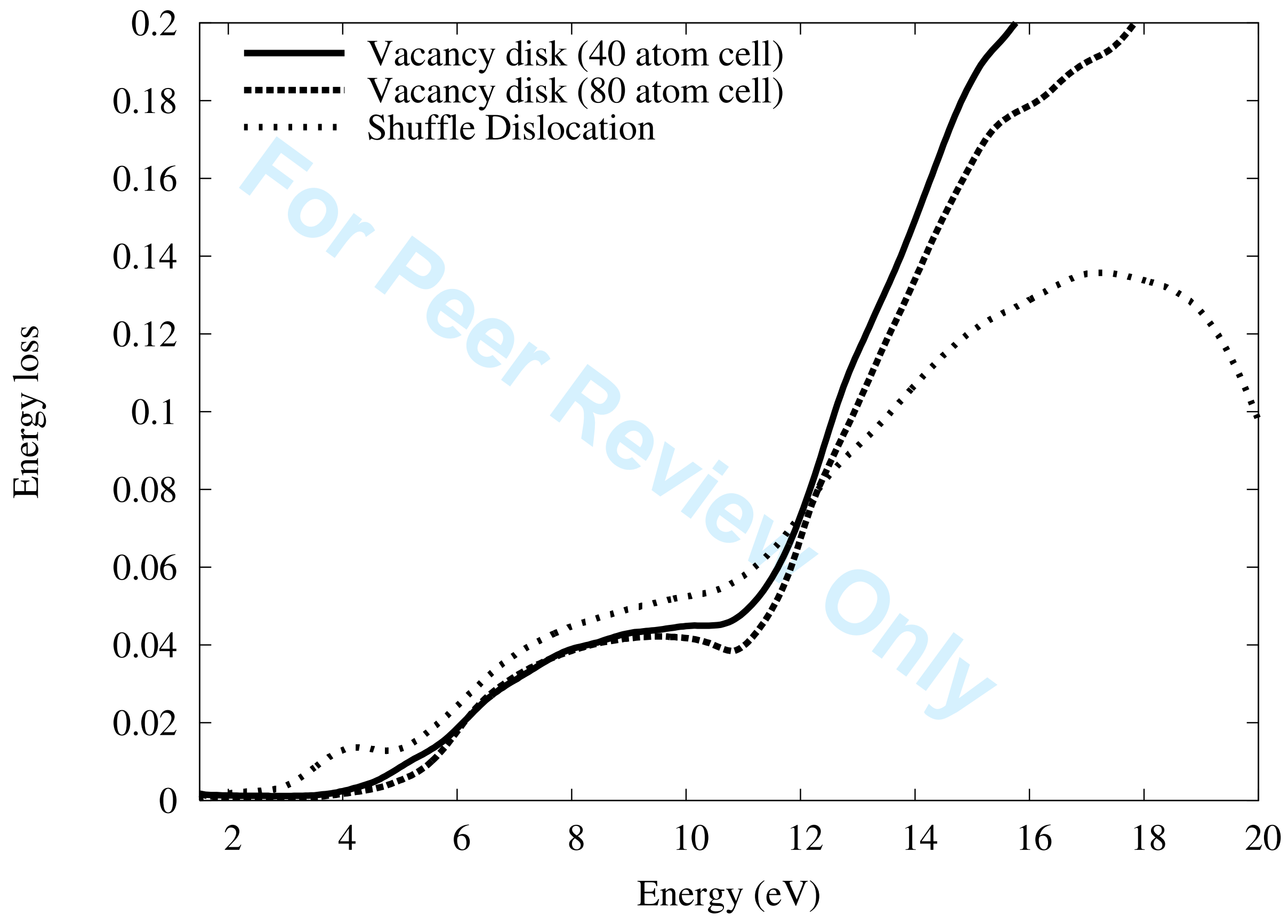

http://mc.manuscriptcentral.com/pm-pml 


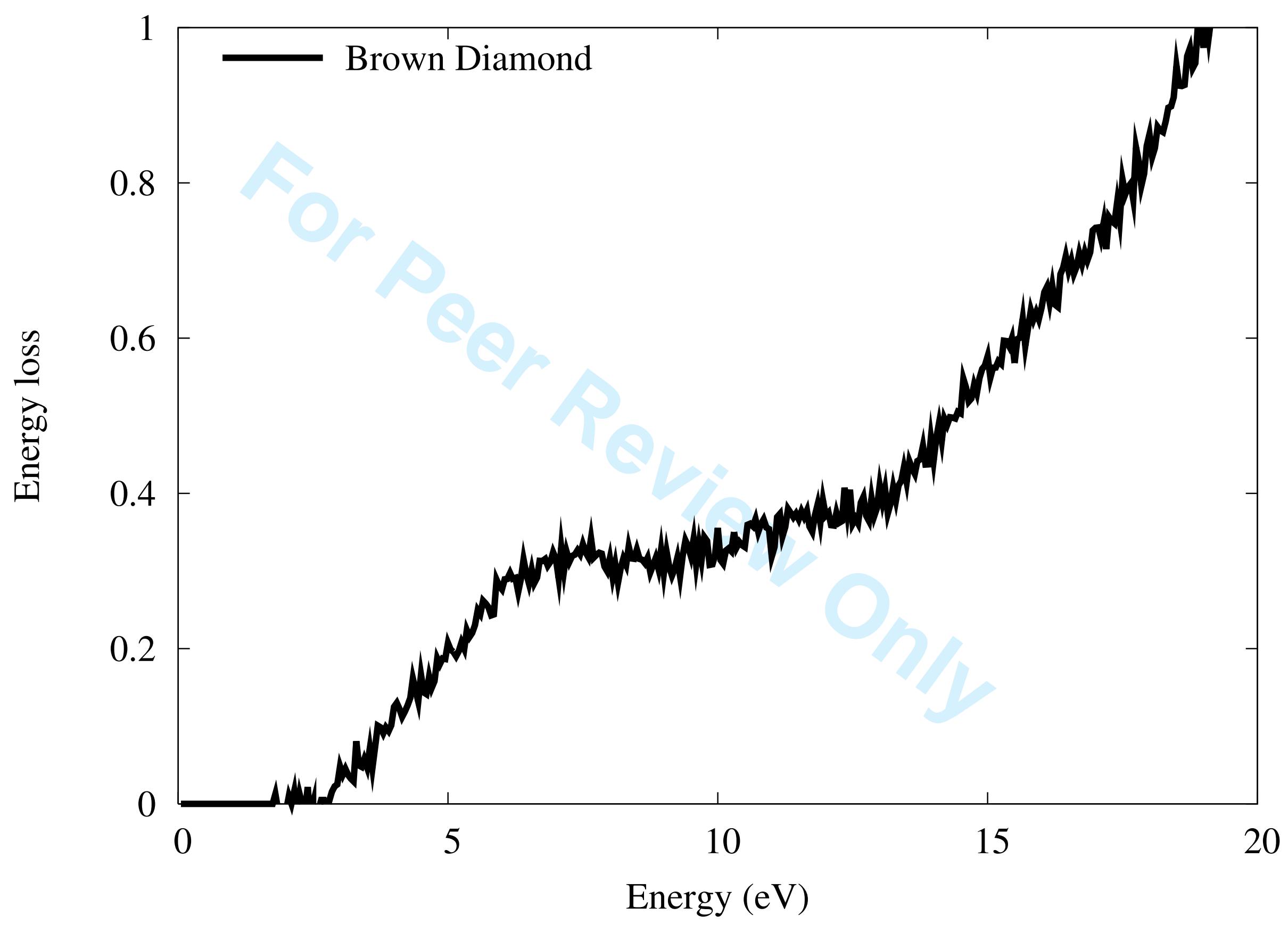

http://mc.manuscriptcentral.com/pm-pml 


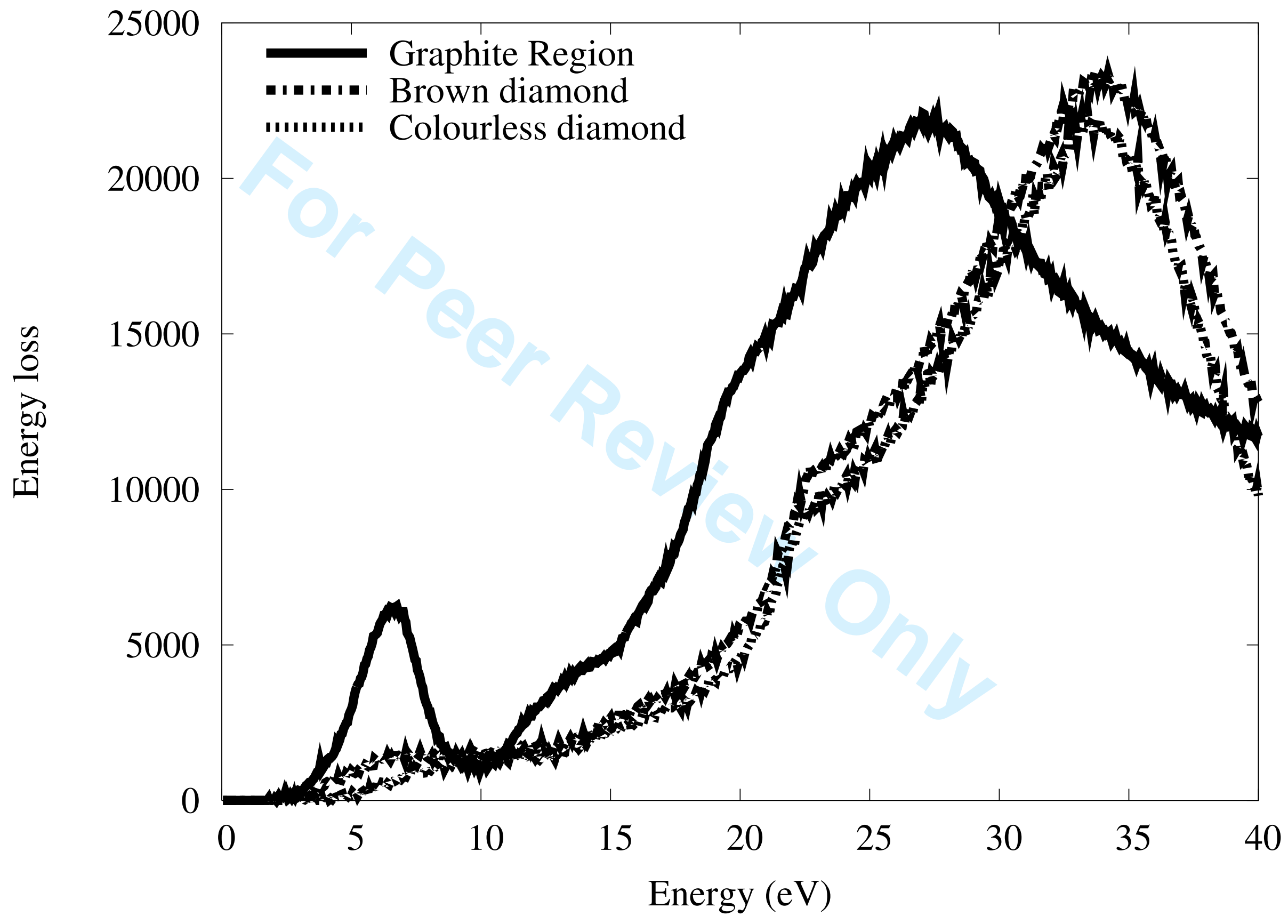

http://mc.manuscriptcentral.com/pm-pml 


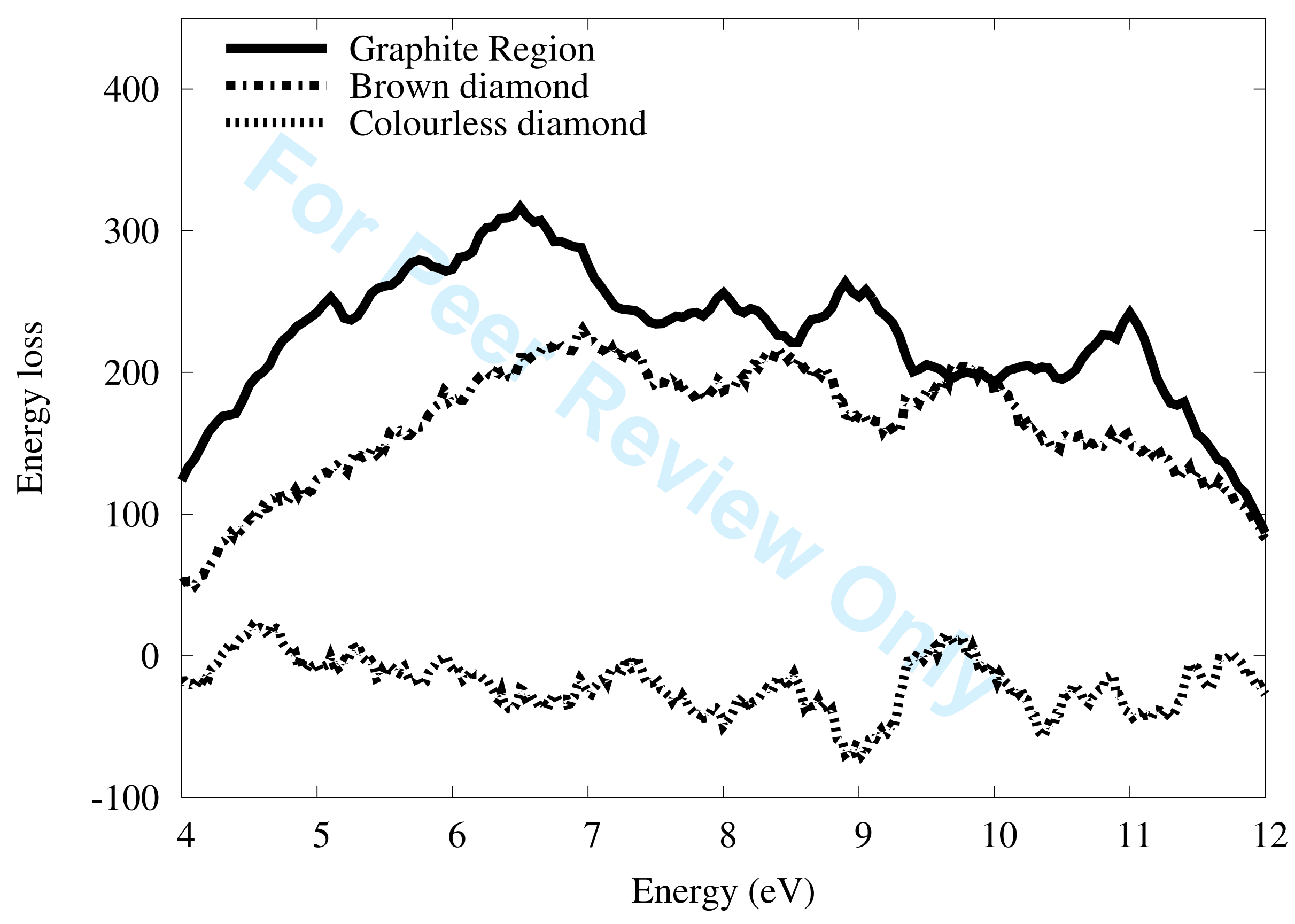

http://mc.manuscriptcentral.com/pm-pml 


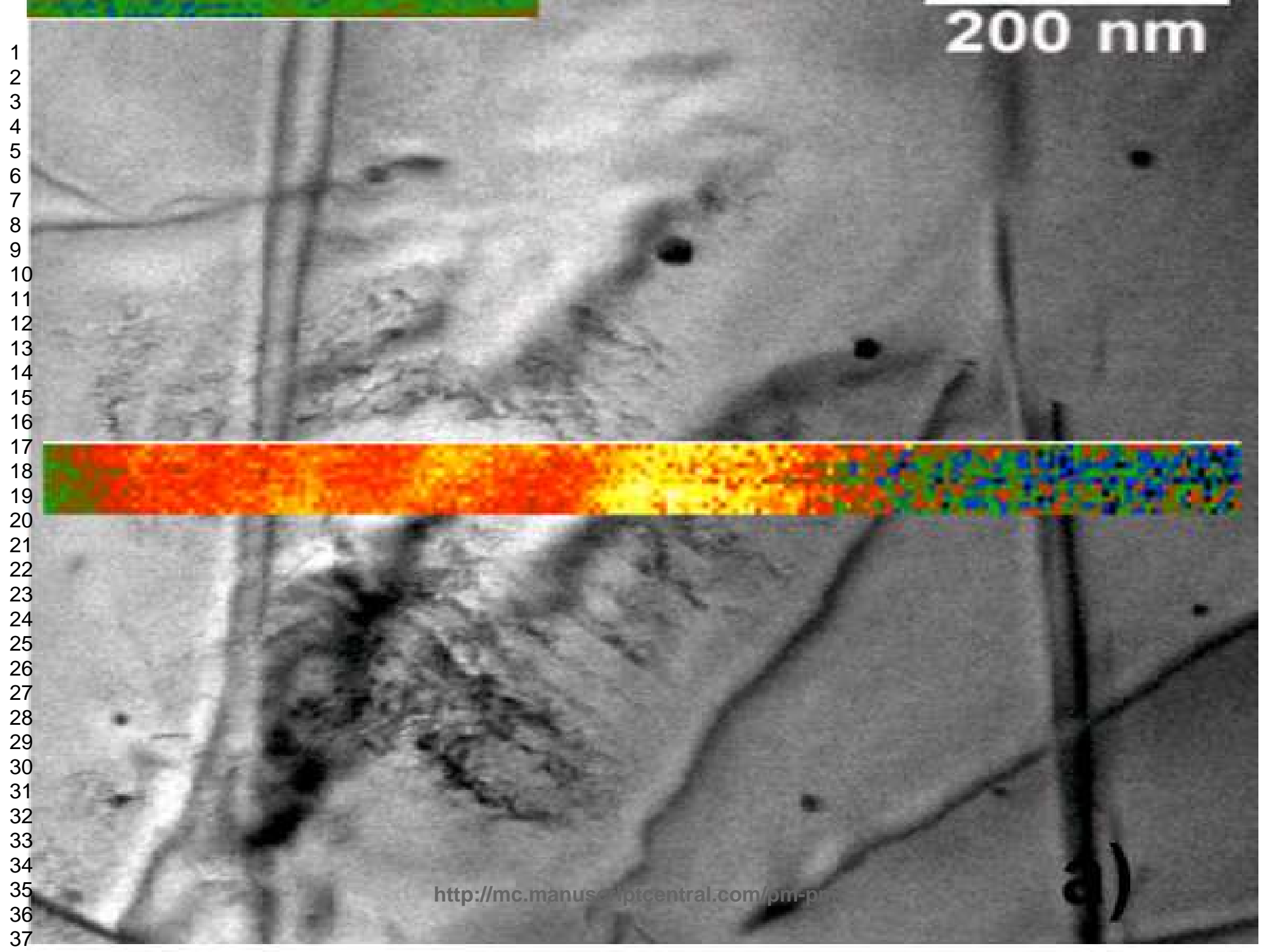
37 38 


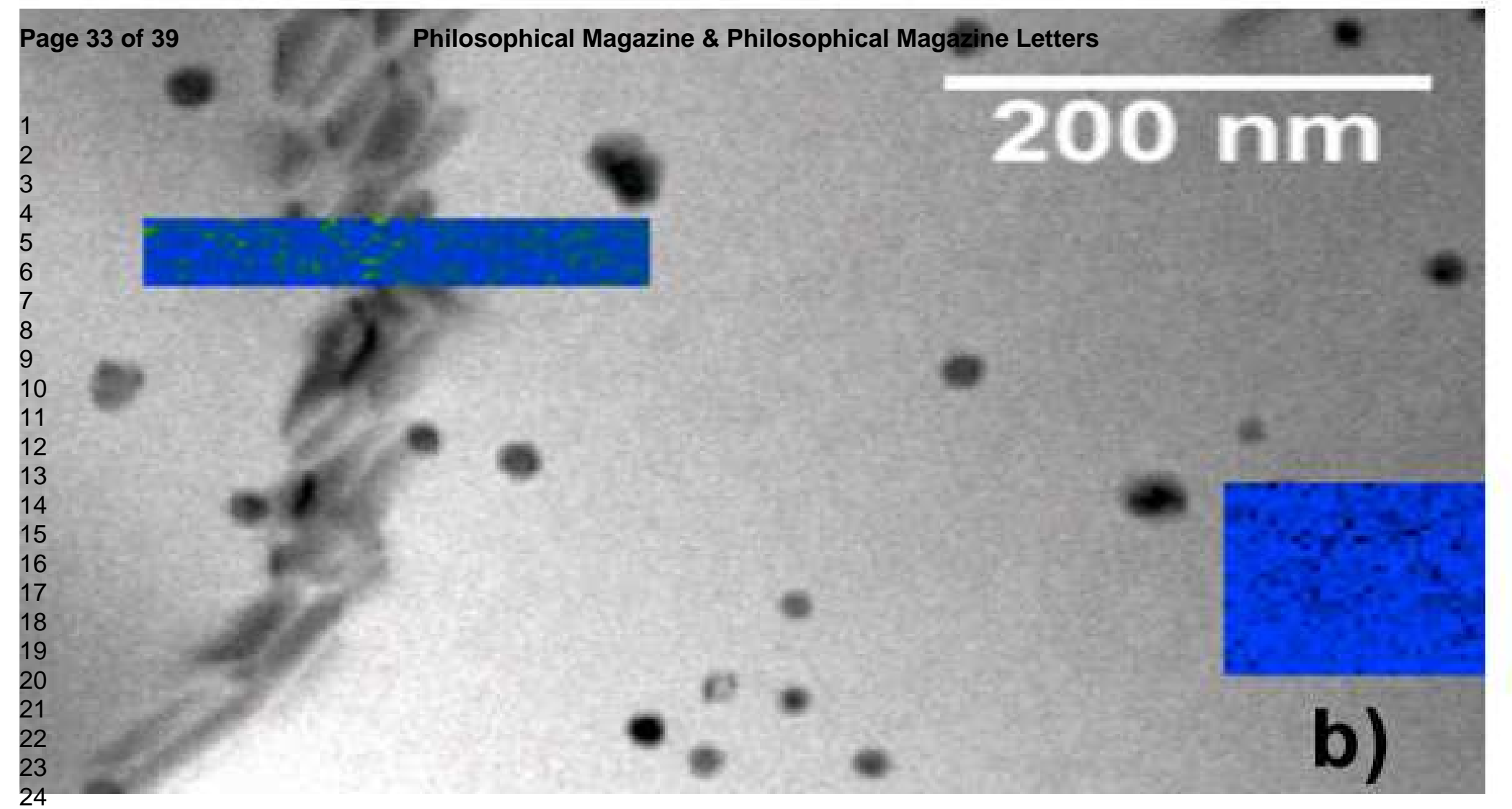



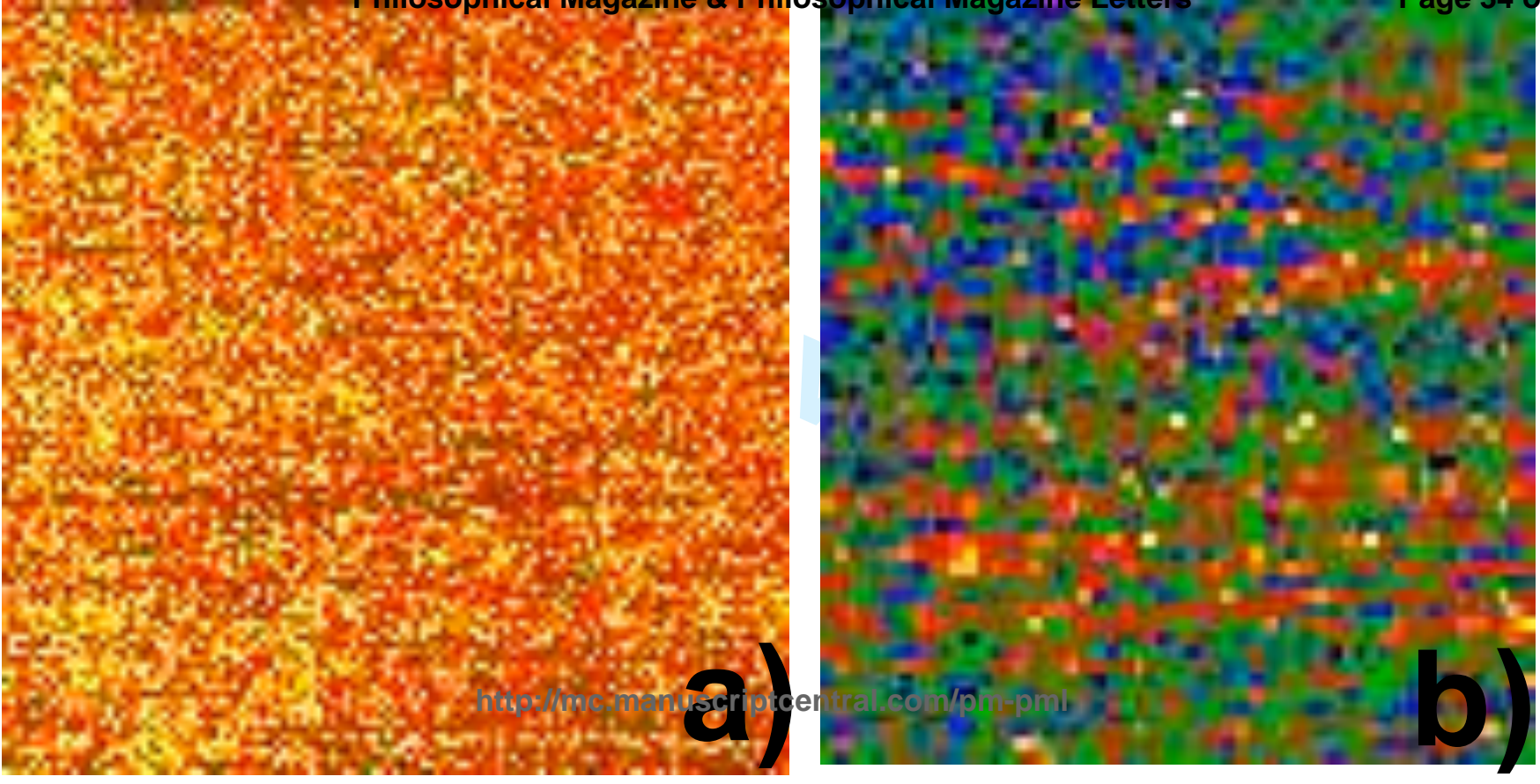

ad

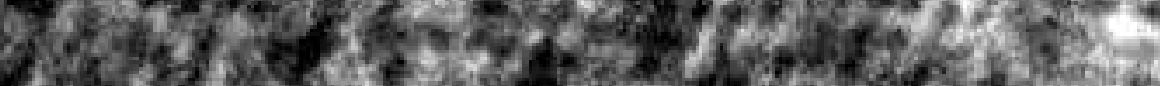

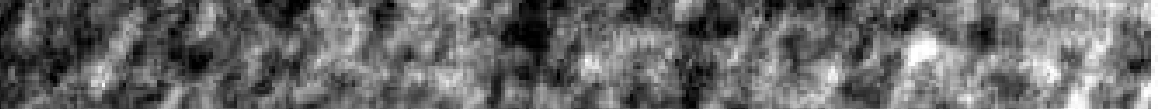

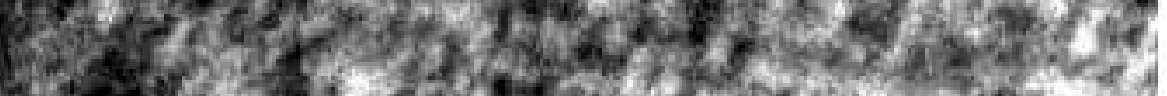

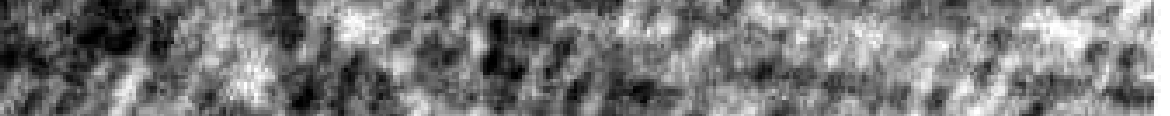

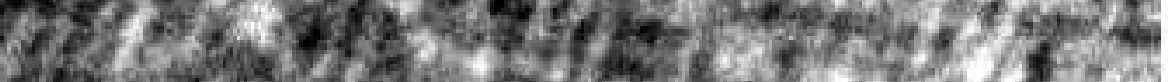

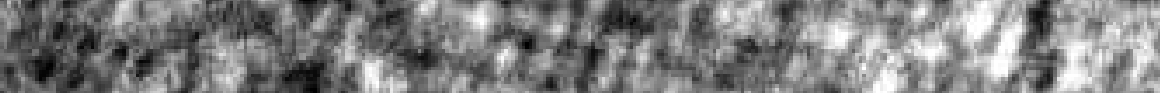

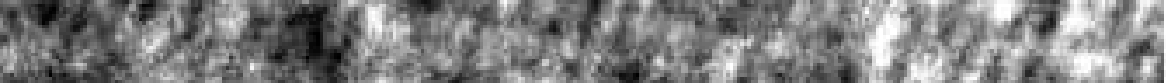

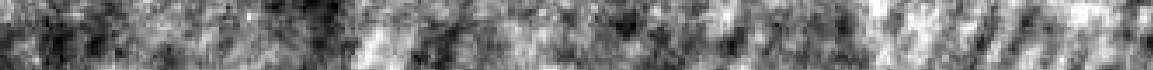

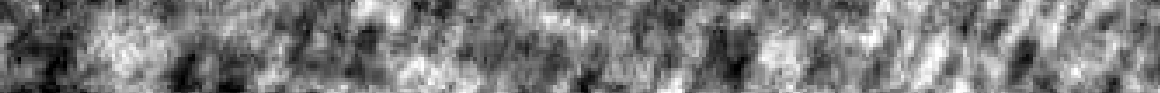

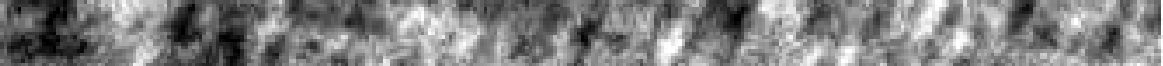

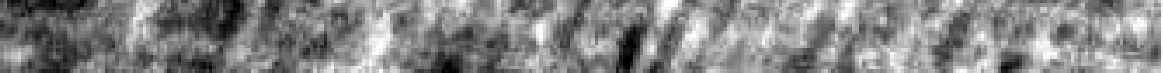

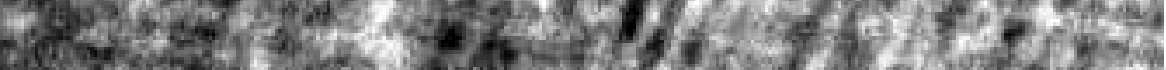

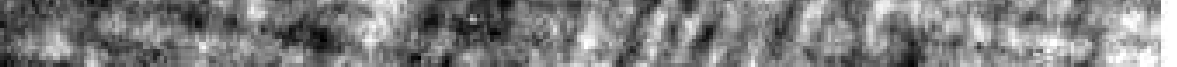

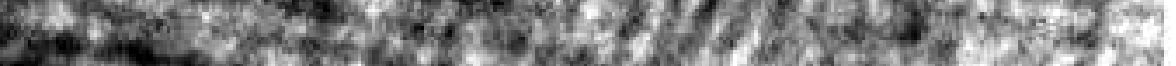

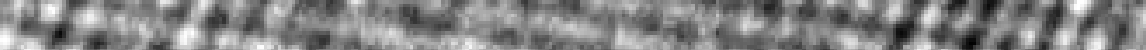

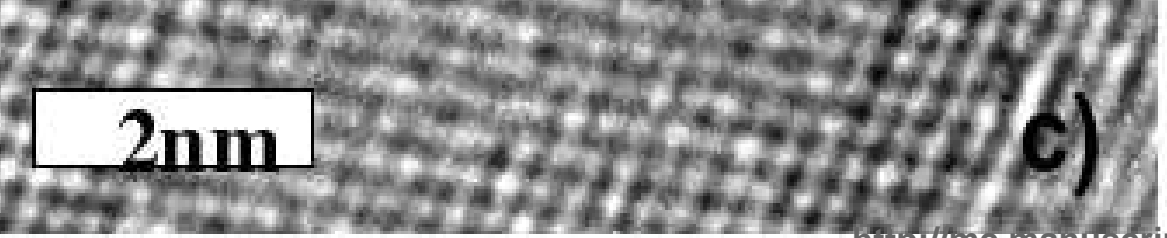
http://mc.manuscriptcentral.com $/ \mathrm{pm}-\mathrm{pm}$

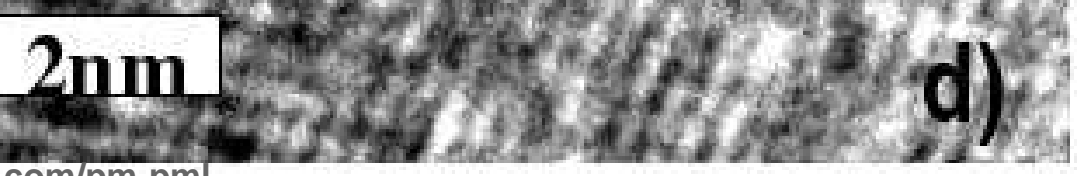




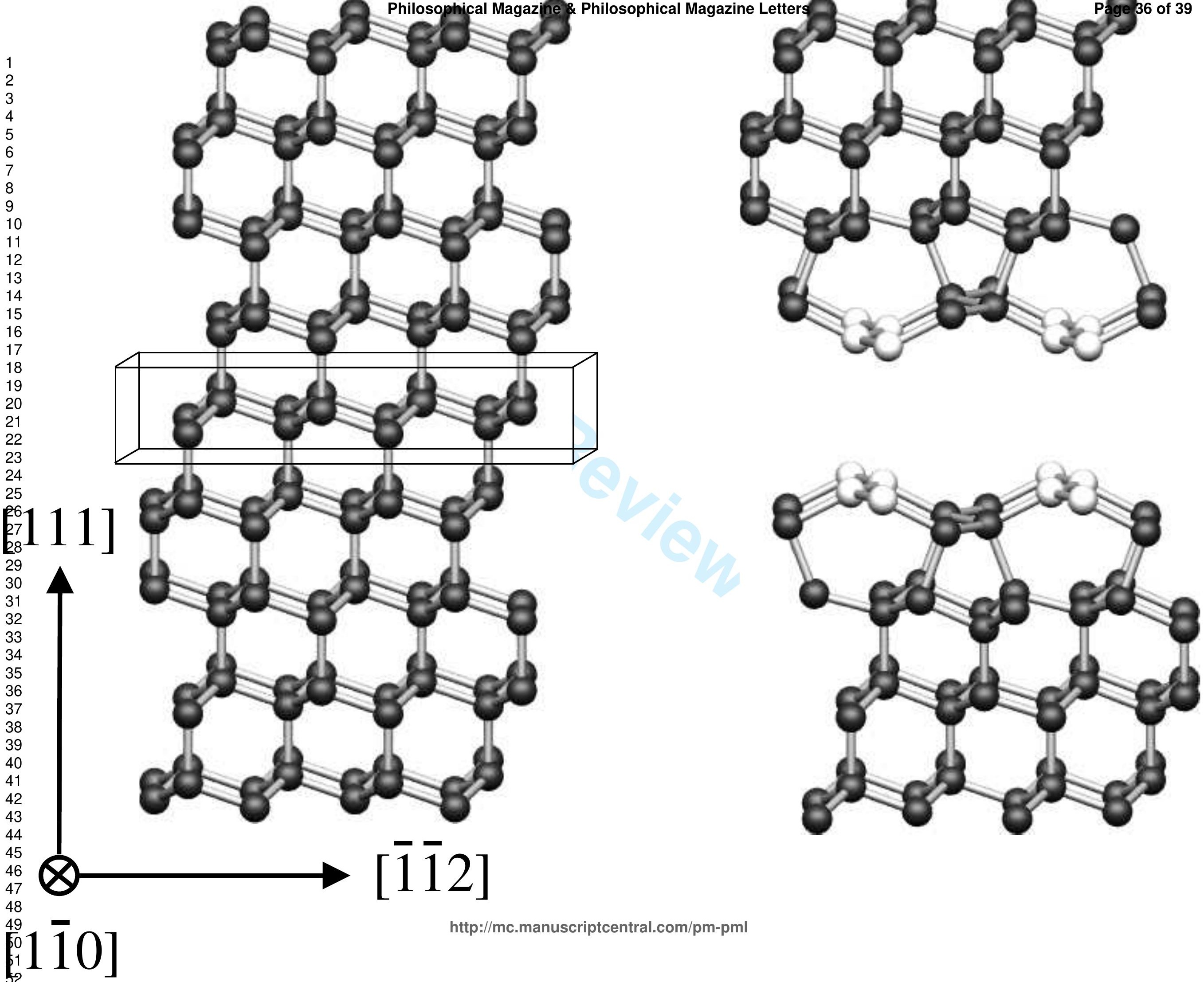




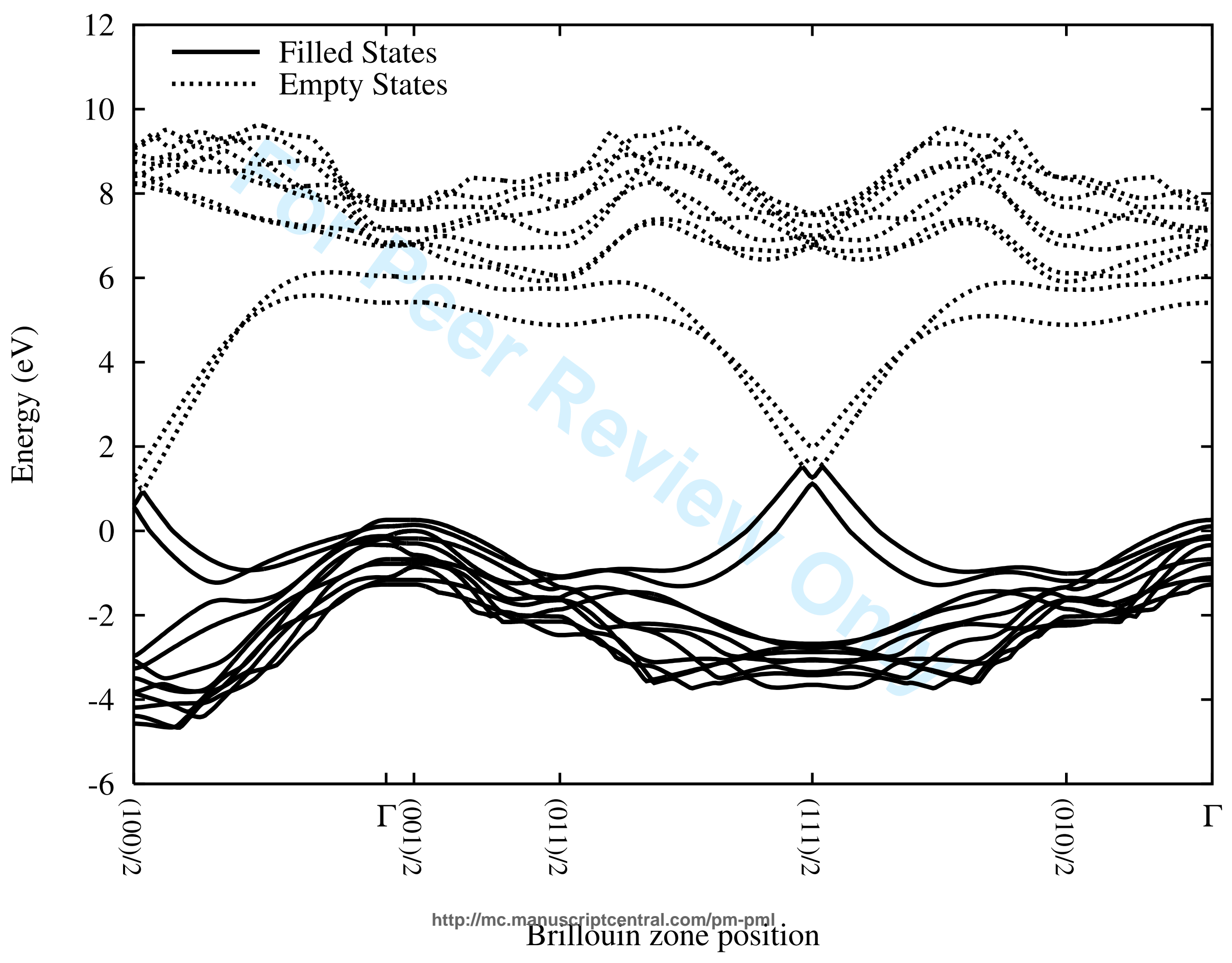




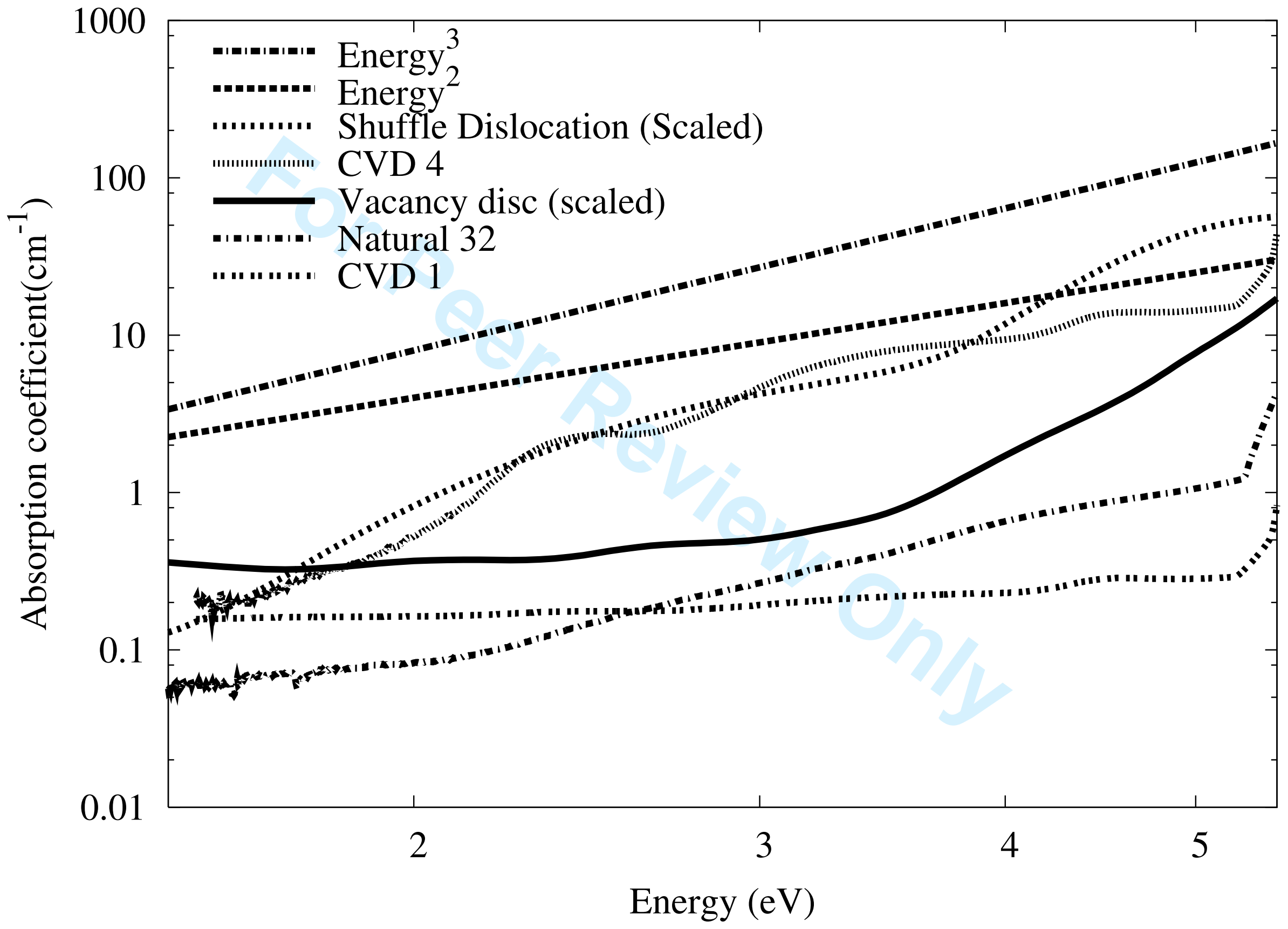




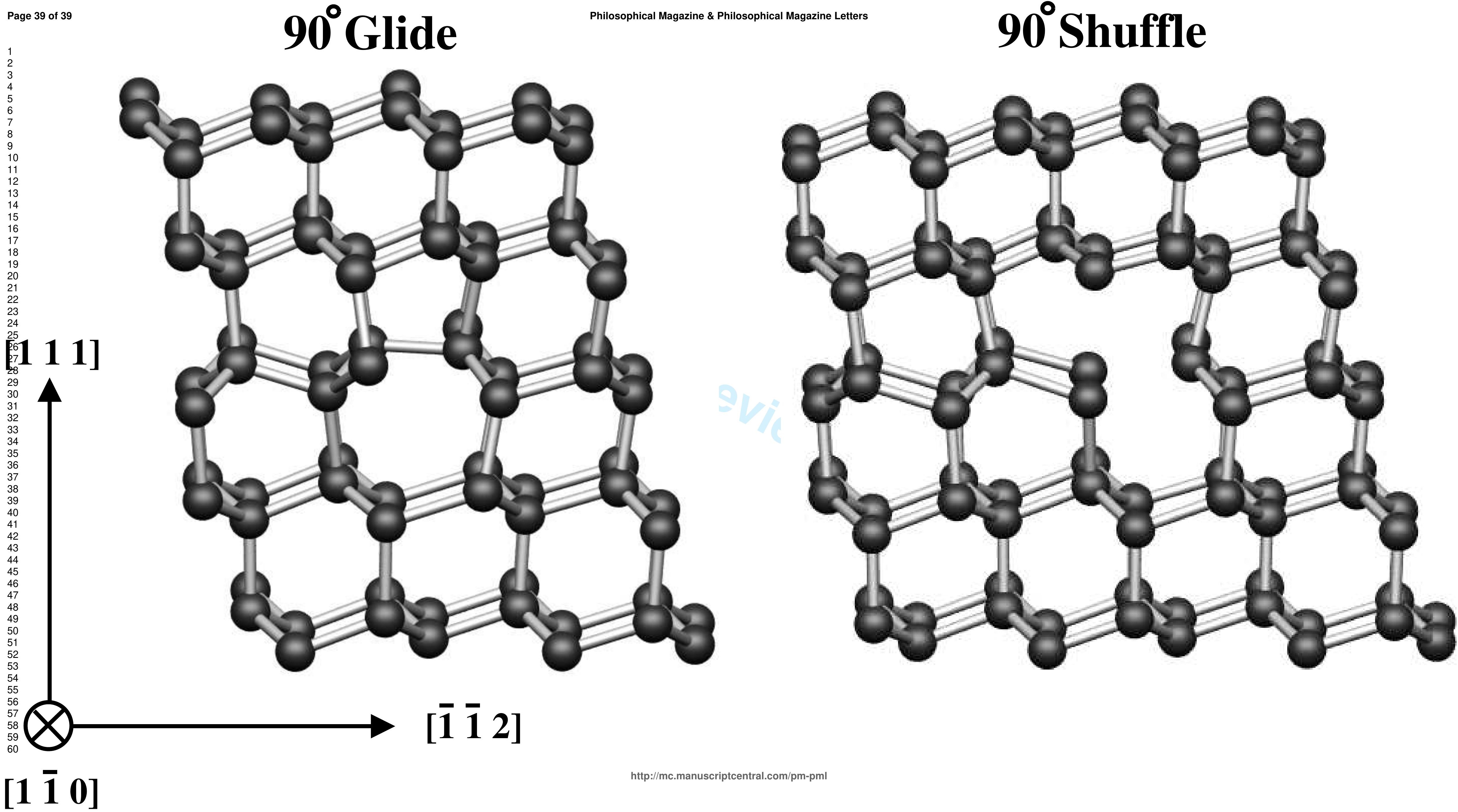

